Published in final edited form as:

Nat Neurosci. 2020 April ; 23(4): 487-499. doi:10.1038/s41593-019-0582-1.

\title{
Preservation of a remote fear memory requires new myelin formation
}

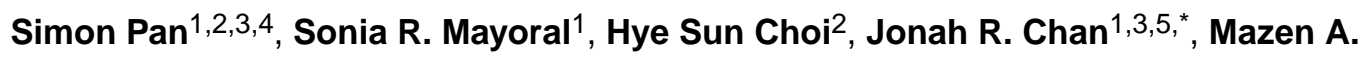 \\ Kheirbek $^{2,3,5,6,7,{ }^{*}}$ \\ ${ }^{1}$ Department of Neurology, University of California, San Francisco, San Francisco, CA, USA. \\ ${ }^{2}$ Department of Psychiatry, University of California, San Francisco, San Francisco, CA, USA. \\ ${ }^{3}$ Neuroscience Graduate Program, University of California, San Francisco, San Francisco, CA, \\ USA.
}

${ }^{4}$ Medical Scientist Training Program, University of California, San Francisco, San Francisco, CA, USA.

${ }^{5}$ Weill Institute for Neuroscience, University of California, San Francisco, San Francisco, CA, USA.

${ }^{6}$ Kavli Institute for Fundamental Neuroscience, University of California, San Francisco, San

Francisco, CA, USA.

${ }^{7}$ Center for Integrative Neuroscience, University of California, San Francisco, San Francisco, CA, USA.

\begin{abstract}
Experience-dependent myelination is hypothesized to shape neural circuit function and subsequent behavioral output. Using a contextual fear memory task in mice, we demonstrate that fear learning induces oligodendrocyte precursor cells to proliferate and differentiate into myelinating oligodendrocytes in the medial prefrontal cortex. Transgenic animals that cannot form new myelin exhibit deficient remote, but not recent, fear memory recall. Recording population calcium dynamics by fiber photometry, we observe that the neuronal response to conditioned context cues evolves over time in the medial prefrontal cortex, but not in animals that cannot form new myelin. Finally, we demonstrate that pharmacological induction of new myelin formation with clemastine fumarate improves remote memory recall and promotes fear generalization. Thus, bidirectional
\end{abstract}

\footnotetext{
Reprints and permissions information is available at www.nature.com/reprints.

*Correspondence and requests for materials should be addressed to J.R.C. or M.A.K. jonah.chan@ucsf.edu; mazen.kheirbek@ucsf.edu.

Author contributions

S.P. conceptualized the project. S.P. designed and performed all the experiments. M.A.K. and J.R.C. provided technical expertise, funding and advice. S.P., M.A.K. and J.R.C. drafted and edited the manuscript. S.P. wrote all of the code for photometry signal processing and analyses, performed the quantification and data analyses and prepared the figures. S.R.M. prepared the electron microscopy samples and performed image quantification. H.S.C. assisted with histological preparations and quantification.

Competing interests

The authors declare no competing interests.

Extended data is available for this paper at https://doi.org/10.1038/s41593-019-0582-1.

Supplementary information is available for this paper at https://doi.org/10.1038/s41593-019-0582-1.

Publisher's note Springer Nature remains neutral with regard to jurisdictional claims in published maps and institutional affiliations.
} 
manipulation of myelin plasticity functionally affects behavior and neurophysiology, which suggests that neural activity during fear learning instructs the formation of new myelin, which in turn supports the consolidation and/or retrieval of remote fear memories.

The formation of enduring memories requires anatomical substrates that are both plastic (to represent new experiences) and perseverant (to preserve lasting representations of those experiences). Although the majority of myelination occurs during postnatal development, oligodendrocyte precursor cells (OPCs) persist in the adult brain and continually proliferate and differentiate to produce myelinating oligodendrocytes (OLs) throughout life ${ }^{1-6}$. OPCs exhibit excitatory postsynaptic potentials in response to neurotransmitter release $\mathrm{e}^{7,8}$ and, in adult rodents, optogenetic or chemogenetic stimulation of neurons is sufficient to induce proliferation, differentiation and maturation of cortical OPCs into myelinating $\mathrm{OLs}^{9-11}$. Naturalistic experiences such as motor learning, social isolation, environmental enrichment and sensory deprivation are similarly able to regulate OPC proliferation and differentiation, which raises the possibility that experience-induced myelin formation may represent an alternative, or additional, modality of structural remodeling during learning that is distinct from synaptic plasticity ${ }^{4,12-17}$. Notably, studies monitoring the dynamics of OL populations over time through pulse-chase labeling or longitudinal in vivo two-photon imaging report that myelinating OLs are extraordinarily stable after formation, which uniquely positions new myelin formation as a durable substrate to support lifelong memories ${ }^{1,3,18}$.

Myelination confers profound changes in the biophysical and molecular properties of axons by increasing transmembrane resistance, decreasing membrane capacitance and coordinating architectural rearrangements of voltage-gated ion channels to enable rapid saltatory conduction $^{19}$. Although it is well appreciated that myelination increases conduction velocity across individual axons, how this translates to computations at the level of neural circuits and their subsequent behavioral outputs is poorly understood ${ }^{20}$. Inhibition of new myelin formation before presenting adult mice to a complex wheel with missing rungs impairs motor learning, thus demonstrating that new myelin formation is important for skilled learning ${ }^{15,17}$. However, it is not known what abnormalities in circuit activity underlie these behavioral deficits or whether these findings extend to other learning modalities, such as episodic and emotional memory, which are very distinct in their anatomical basis, mnemonic content and clinical significance.

Fear memory is a highly evolutionarily conserved behavior that is critical for evaluating and responding to threatening situations and can become pathologically maladaptive in neuropsychiatric diseases such as post-traumatic stress disorder (PTSD) ${ }^{21,22}$. Over time, fear memories become consolidated via a process involving the reorganization of neuronal networks into a stable memory trace that can be recalled at least several weeks later; this process is referred to as remote memory. While a number of studies have identified putative circuits that are involved in the consolidation of remote memories ${ }^{23-27}$, the cellular and synaptic mechanisms through which remote memories are consolidated, maintained and retrieved remain an active area of investigation ${ }^{28,29}$. We found that contextual fear conditioning, a Pavlovian learning paradigm in which an association is formed between a neutral context and an aversive foot shock, induced OPCs to proliferate and mature into 
myelinating OLs in the medial prefrontal cortex (mPFC). To examine the functional significance of this new myelin formation in fear learning, we assessed fear memory in transgenic mice that are unable to form new OLs and found that remote, but not recent, fear memory recall was impaired in the absence of oligodendrogenesis. Furthermore, increasing new myelin formation through chronic administration of the pro-myelinating compound clemastine fumarate preserved the retrieval of remote fear memory. In both cases, immediate early gene expression in fear-associated brain regions paralleled the changes in learning performance. To probe this neurophysiological deficit with greater temporal resolution, we recorded population calcium dynamics in freely behaving mice via fiber photometry and observed altered prefrontal activity in the absence of new oligodendrogenesis during remote, but not recent, memory recall. Collectively, these findings suggest a reciprocal relationship in which learning experience induces new myelin formation, which in turn supports the neural circuits of remote fear memories.

\section{Results}

\section{Fear learning experience induces OPC proliferation in the mPFC.}

To determine whether fear learning induces OPC proliferation and differentiation, we injected wild-type 8-week-old male C57BL/6J mice with 5-ethynyl-2' -deoxyuridine (EdU), a thymidine analog that is incorporated by actively proliferating cells, and immediately subjected the mice to contextual fear conditioning (Fig. 1a). At $24 \mathrm{~h}$ post-conditioning, conditioned mice froze in the conditioning context, which was indicative of successful learning of the shock-context association (Fig. 1b). The mice were then perfused for subsequent histological analysis. We detected an increase in the number of total EdU ${ }^{+}$cells and OPCs positive for both EdU and oligodendrocyte transcription factor 2 (Olig2) in the $\mathrm{mPFC}$ of conditioned mice compared with home-cage controls (Fig. 1c-f). As novel context exposure and the foot-shock stress can conceivably induce these changes, we included two additional control groups in which the mice either received a context-only exposure (no shock) or were shocked immediately after being put in the chamber and promptly removed (immediate shock). Both groups of mice displayed low levels of freezing during the retrieval session (Fig. 1b), which indicates the absence of learning, and correspondingly showed no changes in the number of EdU ${ }^{+}$Olig2 ${ }^{+}$cells (Fig. 1c,d). These results indicate that fear learning experience, independently of novel context exposure or foot-shock stress, induces a rapid increase in OPC proliferation in the $\mathrm{mPFC}$.

\section{Fear learning experience induces OPC maturation into myelinating OLs in the MPFC.}

Next, we determined whether this brisk proliferation of OPCs culminated in their maturation into myelinating OLs. We conducted an extended experiment in which the mice were injected with EdU for five consecutive days and then fear conditioned, with their freezing responses measured during retrieval sessions occurring $24 \mathrm{~h}$ and 30 days post-conditioning (Fig. 2a). Freezing responses were maintained over the course of the experiment (Fig. 2b), and the number of EdU ${ }^{+}$cells colocalizing with aspartoacylase (ASPA), a marker for differentiated OLs, was increased in the mPFC with no change in the total amount of EdU ${ }^{+}$Olig $2^{+}$cells (Fig. $2 \mathrm{c}-\mathrm{e}$ ). In addition to the $\mathrm{mPFC}$, we examined the dorsal hippocampus (dHPC) and the ventral hippocampus (vHPC), the anterior cingulate cortex (ACC) and the 
basolateral amygdala (BLA), brain regions canonically associated with fear learning, and detected an increase in OPC proliferation but not maturation in the BLA (Extended Data Fig. 1a-d,i, j). Subregional analysis of proliferation and maturation in the $\mathrm{mPFC}$ revealed no differences between the prelimbic (PL) and infralimbic (IL) cortices (Extended Data Fig. 1a-d). Finally, we performed electron microscopy 30 days post-conditioning and found that fear learning experience significantly increased the density of myelinated axons in the mPFC of fear-conditioned animals compared with home-cage controls (Fig. 2f-h).

\section{OPC maturation into myelinating OLs occurs over several weeks in adult gray matter.}

To examine the timing of OL maturation in adult gray matter, we quantified the density of $\mathrm{EdU}^{+} \mathrm{ASPA}^{+}$cells and cells positive for both green fluorescent protein (GFP) and myelin basic protein (MBP) in the mPFC, the BLA and the dHPC of taumGFPloxP/loxP;NG2CreERT + mice that were injected with EdU for five consecutive days, fear conditioned and then perfused after their 7-, 14- or 30-day retrieval sessions (Fig. 3a). In this line, membranebound GFP adjacent to a loxP stop codon is expressed under the Tau (also known as Mapt) promoter. NG2CreERT is an inducible Cre line specific to OPCs, while the Tau promoter is active in OLs but not OPCs. Thus, recombined OPCs that successfully differentiate into OLs are exclusively visualized by the expression of GFP. We can further distinguish mature, myelinating OLs from immature, non-myelinating OLs through the presence of distinctive myelin internodes that colocalize with MBP (Fig. 3b,c). We observed that the density of $\mathrm{EdU}^{+} \mathrm{ASPA}^{+}$cells and $\mathrm{GFP}^{+} \mathrm{MBP}^{+}$cells was virtually zero in animals 7 days post-tamoxifen and post-EdU treatment, but increased significantly at 14 and 30 days. Conversely, the density of $\mathrm{GFP}^{+} \mathrm{MBP}^{-}$pre-myelinating OLs remained similar across time (Fig. 3d,e; Extended Data Fig. 1e-g).

Next, we analyzed the colocalization of ASPA, GFP and MBP in tau$m G F P^{\text {loxP/loxP }} ; N G 2 C r e E R T^{+}$animals perfused at 30 days and found that $\mathrm{ASPA}^{+} \mathrm{GFP}^{+}$cells were exclusively $\mathrm{MBP}^{+}$OLs with myelinating morphology. Conversely, $\mathrm{GFP}^{+} \mathrm{MBP}^{+} \mathrm{OLs}$ with myelinating morphology were exclusively ASPA ${ }^{+}$(Fig. 3f,g; Extended Data Fig. 1h), which indicates that ASPA is a specific marker for mature, myelinating OLs. These data suggest that although OPC proliferation and differentiation can occur rapidly, maturation and compact myelin formation in adult gray matter takes place over a prolonged timescale of several weeks. Importantly, the validation of ASPA as a marker of mature, myelinating OLs signifies that the increase in $\mathrm{EdU}^{+} \mathrm{ASPA}^{+}$cells in the mPFC that we observed in response to fear learning (Fig. 2c-e) was indicative of new myelin formation.

\section{Inhibition of new myelin formation impairs remote fear memory recall.}

As previously reported, conditional deletion of the transcription factor Myrf in OPCs arrests their differentiation and prevents the expression of myelin structural genes ${ }^{15,17,30}$. Administration of tamoxifen to adult $M y r f^{d o x P / l o x} P$ animals crossed with the $N G 2 C r e E R T$ line therefore results in the inhibition of oligodendrogenesis and new myelin formation while preserving existing myelinated circuits. We confirmed that this approach inhibits new myelin formation by examining the mPFC of MyrtoxP/loxP ;tau-mGFPloxP/loxP $; N G 2 C r e E R T$ ${ }^{+/-}$and $M y r f^{\text {foxP/+ }}$;tau-mGFPloxP/loxP;NG2CreERT ${ }^{+}$littermates. At 51 days post-tamoxifen

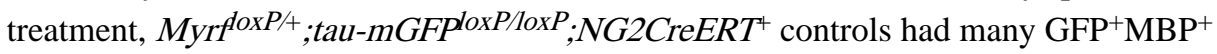


mature OLs with distinctive myelinating morphology, whereas the mPFC of MyrfoxP/loxP, ;au-mGFPloxP/loxP; NG2CreERT ${ }^{+/-}$animals, with both copies of Myrf deleted, contained largely $\mathrm{GFP}^{+} \mathrm{MBP}^{-}$pre-myelinating OLs (Fig. 4c,d). The total density of $\mathrm{ASPA}^{+}$ cells and mean MBP fluorescence were identical between genotypes, which indicates that preexisting myelinating OLs were unaffected by Myrf deletion (Extended Data Fig. 2a-d).

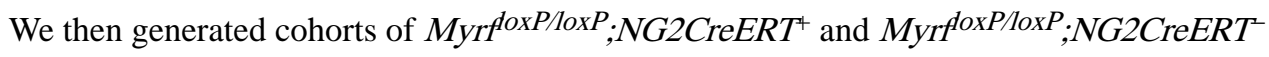
littermate controls (hereafter referred to as $M y r f$ icKO and Cre-negative controls, respectively) and subjected them to contextual fear conditioning 3 weeks after five consecutive days of daily tamoxifen administration, and then assessing their freezing responses at recent and remote time points (Fig. 4a; Extended Data Fig. 3a). While both Crenegative and MyrficKO animals exhibited high levels of freezing during recent retrieval sessions, context-elicited freezing responses in Myrf icKO mice declined over time and diverged from controls at 30 days post-conditioning, which indicates a specific deficit in remote memory (Fig. 4e; Extended Data Fig. 3e) with no differences detected between male and female animals (Extended Data Fig. 3k-1).

To rule out the possibility that inhibition of basal myelin formation during the 3 weeks before conditioning has an effect on fear learning, we conducted identical fear conditioning experiments whereby we instead administered tamoxifen either 5 days preconditioning or just after $24 \mathrm{~h}$ of recall to solely inhibit new myelin formation during the consolidation period (Fig. 4b; Extended Data Fig. 3b,c). These Myrf icKO animals similarly exhibited a freezing deficit 30 days post-conditioning, which suggests that it is new myelin formation during consolidation that is important for remote memory (Fig. 4f; Extended Data Fig. 3f,g). We then reconditioned these animals by administering foot shocks in the original conditioning context 31 days post-conditioning. Both Myrf icKO and Cre-negative control animals exhibited high levels of freezing $24 \mathrm{~h}$ after reconditioning, which demonstrates that there is no generalized impairment of freezing in MyrficKO animals at remote time points (Fig. 4g). Finally, to eliminate the possibility that prolonged inhibition of myelin formation could have nonspecific effects on memory recall, we administered tamoxifen to a separate cohort of Myrf icKO mice 7 weeks before conditioning (Extended Data Fig. 3d), a period equivalent to the length of tamoxifen recombination experienced by the cohort represented in the initial experiment at 30 days post-conditioning (Fig. 4a; Extended Data Fig. 3a). If prolonged tamoxifen recombination alone could lead to impaired fear memory recall, then freezing deficits should be observed even $24 \mathrm{~h}$ post-conditioning in this cohort. This was not the case (Extended Data Fig. 3h), thereby indicating that the recall deficits observed in Myrf icKO animals are specific to remote recall.

Additionally, we measured anxiety-like behaviors in the open field test (OFT) and elevated plus maze (EPM) in MyrficKO and Cre-negative control animals 3 weeks post-tamoxifen administration. Both genotypes displayed similar total distance traveled and time spent in the periphery of the OFT or closed arms of the EPM, which suggests that differences in freezing behavior are not driven by variances in innate anxiety or locomotor activity (Extended Data Fig. 4a-e). As inhibition of OPC differentiation may lead to the death of arrested cells and subsequent inflammation that could perturb circuit function, we quantified 
microglial density in the mPFC of Myrf icKO and Cre-negative animals and found no differences in Iba1 ${ }^{+}$cell density between genotypes (Extended Data Fig. 2e,f).

\section{Immediate early gene expression following remote memory recall is impaired in the absence of new myelin formation.}

To assess neuronal activity in response to remote memory recall, we perfused Myrf icKO and Cre-negative control animals 90 min after the 30-day retrieval session and conducted histological analysis of Fos induction in a number of fear-associated brain regions. We found that the density of $\mathrm{Fos}^{+}$cells was significantly decreased in the prelimbic cortex (PL) and infralimbic cortex (IL) of the mPFC, the ACC, the basal and lateral amygdala of the BLA, the nucleus reuniens and the dentate gyrus (DG) and CA3 of the dHPC and vHPC in Myrf icKO animals compared with Cre-negative controls (Fig. 5a,c-e; Extended Data Fig. 3i). In addition, the degree of Fos induction displayed a linear relationship to individual freezing at 30 days post-conditioning (Fig. 5g-j). Notably, Fos induction was similar across genotypes in home-cage controls at 30 days post-conditioning (Fig. 5b) and following recent memory retrieval at $24 \mathrm{~h}$ post-conditioning (Fig. 5f). Fos $^{+}$cell density was also similar in a non-fearassociated brain region, the somatosensory cortex (SSC), across genotypes (Fig. 5a). This indicates that the physiological response of neurons within the fear memory network is specifically impaired in the absence of myelin formation during remote memory recall.

\section{Population calcium dynamics in the MPFC are altered in the absence of new myelin formation.}

To relate neuronal activity to freezing behavior in Myrf icKO animals and Cre-negative controls with high temporal resolution, we performed fiber photometry recordings in freely behaving mice during contextual fear conditioning and retrieval sessions (Fig. 6a; Extended Data Fig. 5a,b). As we detected no difference in Fos induction between the PL and IL of the mPFC (Extended Data Fig. 3i,j), we injected adeno-associated viral (AAV) vectors expressing the calcium indicator GCaMP6f and implanted optical fibers at the PL-IL border of the mPFC (Fig. 6b,c). This targeting effectively records from the IL, where suppression of firing of $\mathrm{mPFC}$ units in response to conditioned cues has previously been reported ${ }^{31,32}$. Successful virus and fiber delivery were verified through post hoc histology, and minimal microglial infiltration was observed in chronic implants (Extended Data Fig. 5f,g).

Following recovery from the surgery, animals were given tamoxifen for 5 days, rested for 3 weeks and then subjected to contextual fear conditioning (Fig. 6a). Both control and Myrf icKO animals displayed robust neuronal responses to foot shock in the mPFC, with no difference in shock-evoked activity between genotypes (Fig. 6d-e,h). To determine whether recall-induced neuronal activity was altered in the absence of new myelin formation, we aligned photometry signals to behavioral footage during retrieval sessions and analyzed the changes in mPFC activity during transitions from mobility to freezing (Fig. 6f,g). In line with previous reports in which electrophysiological recordings of IL units were obtained $^{31,32}$, we found that at $24 \mathrm{~h}$ post-conditioning, mPFC activity was suppressed during fear memory recall in both control and Myrf icKO animals (Fig. 6g,i, Extended Data Fig. $5 \mathrm{c}, \mathrm{d})$. However, we found that prefrontal dynamics associated with fear recall evolved over time in control animals, which exhibited an elevation of mPFC activity during transitions 
from mobility to freezing at 30 days post-conditioning (Fig. 6g,i; Extended Data Fig. 5c,d). This time-dependent change in the recall-associated response was not evident in Myrf icKO animals, which continued to show a suppression of prefrontal activity during freezing transitions at 30 days post-conditioning (Fig. 6g,i; Extended Data Fig. 5c,d). Baseline activity in the home cage before 24-h or 30-day retrieval sessions did not differ between genotypes (Extended Data Fig. 5e). The absence of this temporal evolution of prefrontal dynamics in Myrf icKO animals suggests that active oligodendrogenesis is required for remote fear memory consolidation.

\section{Inducing new myelin formation preserves retrieval of a contextual fear memory.}

Clemastine fumarate is an antimuscarinic compound that was identified as a pro-myelinating agent in high-throughput screening efforts to find therapeutics for demyelinating disease ${ }^{33,34}$. Administration of clemastine to humans and rodents with inflammatory demyelination promotes remyelination and relieves the severity of clinical symptoms ${ }^{35,36}$. Clemastine also promotes myelination and rescues behavioral deficits in mice that are hypomyelinated following either prolonged social isolation or chronic hypoxia ${ }^{36,37}$. To determine whether induction of new myelin formation affects fear memory, we subjected cohorts of wild-type 8-week-old male C57BL/6J mice to contextual fear conditioning while injecting them daily with either clemastine or vehicle from 3 days pre-conditioning to 21 days post-conditioning (Fig. 7a; Extended Data Fig. 6a). We verified that chronic clemastine administration increases new myelin formation under healthy, physiological conditions, as confirmed by observing increased ASPA ${ }^{+}$cell density and mean MBP fluorescence intensity in the mPFC of clemastine-treated mice (Fig. 7b-d).

In contrast to Cre-negative controls in the previous MyrficKO experiments, vehicle controls exhibited decreasing freezing responses over time. We suspected that this discrepancy could be due to habituation from prolonged daily handling and injections; therefore, we tested a separate cohort in which freezing responses between vehicle-injected and home-cage C57BL/6J animals that received no treatment were compared. We found that continuous injections of vehicle alone resulted in a reduction in freezing responses over time compared with home-cage controls (Extended Data Fig. 6d). Nonetheless, clemastine-treated mice displayed persistent and high freezing responses over time, which suggests that increasing new myelin formation stabilizes remote fear memory (Fig. 7e; Extended Data Fig. 6b). We also assessed fear generalization by measuring freezing responses in a context that is similar to the conditioning context, but is either missing or contains a variant of the original context cues (Extended Data Fig. 6a). As previously reported, fear generalization increases with the age of the memory ${ }^{38}$; however, clemastine-treated mice showed a more pronounced increase in generalization than vehicle-treated controls (Extended Data Fig. 6c). Importantly, baseline freezing behavior following remote memory testing was not affected by chronic clemastine administration in home-cage, no shock and immediate shock mice that never learned the fear association (Extended Data Fig. 6a,g-i). To rule out the possibility that a remote memory phenotype is not simply a result of prolonged clemastine treatment, we gave mice clemastine every day for 3 weeks before conditioning and observed normal freezing behavior during recent memory recall (Fig. 7f-g). Finally, we administered vehicle or clemastine injections to Myrf icKO mice and subjected them to the contextual fear learning paradigm (Fig. $7 \mathrm{~h}$ ). In 
the absence of new myelin formation, clemastine-treated Myrf icKO animals appeared identical to vehicle-treated animals (Fig. 7i), which indicates that the effects of clemastine on fear memory are specifically dependent on new myelin formation. Collectively, these results indicate that inducing new myelin formation preserves remote fear memory, but may potentially decrease its precision.

We then perfused vehicle-treated and clemastine-treated animals $90 \mathrm{~min}$ after the 30-day retrieval session for subsequent histological analysis of immediate early gene expression. Fos $^{+}$cell density was increased in clemastine-treated animals in the mPFC, the ACC, the BLA, the nucleus reuniens, the ventral periaqueductal gray and the DG and CA3 of the dHPC and vHPC, but not the SSC (Fig. 8a-c; Extended Data Fig. 6e,f). However, no differences in Fos induction were detected following recent memory recall in mice pretreated with 3 weeks of clemastine or following remote memory recall in home-cage mice that never learned the fear association (Fig. 8d,e). These results suggest that population neuronal activity in response to conditioned cues is increased in fear-associated brain regions following chronic administration of clemastine. Finally, we performed fiber photometry recordings in vehicle-treated animals and clemastine-treated animals during remote memory recall. In contrast to $M y r f$ icKO animals, clemastine-treated mice exhibited increased population activity during freezing bout transitions during remote recall (Extended Data Fig. 61). However, we did not detect any differences between vehicle-treated animals and clemastine-treated animals (Extended Data Fig. 6m), perhaps due to a ceiling effect, an inability for bulk calcium imaging to detect subtle differences in mPFC ensemble activity and/or a nonlinear relationship between myelin content and population dynamics.

\section{Discussion}

In this study, we demonstrated that the generation of new OLs is required for the proper functioning of neural circuits that mediate remote fear memory. This process is specific to remote memory, as Myrf icKO mice appeared identical to Cre-negative control animals at recent time points by many metrics, including freezing behavior, immediate early gene expression and $\mathrm{mPFC}$ population calcium dynamics during both conditioning and recent retrieval sessions. Furthermore, these behavioral and neurophysiological deficits manifest after several weeks, which suggests that it is the formation of new myelin, which occurs over a timescale of weeks as we have described here, and not OL differentiation per se, which occurs over a timescale of hours ${ }^{17}$, that is important for remote fear memory recall. In addition to our electron microscopy analyses, we verified that in the healthy adult brain, ASPA is an exclusive marker for mature, myelinating OLs extruding $\mathrm{MBP}^{+}$processes with characteristic morphology of compact myelin sheaths. Thus, we are able to demonstrate that fear learning induces the formation of bona fide new myelin in the prefrontal cortex.

In two seminal studies, Myrf icKO mice displayed impairments in learning to run on a complex wheel as early as several hours into training. This rapid learning deficit was correlated with the immediate differentiation of OPCs into immature OLs within this same time window ${ }^{15,17}$. In contrast, the onset of deficits we have observed occurs much more slowly, which reflects the time required for compact myelin formation in the adult brain. Learning impairment on a more rapid timescale may conceivably be due to a loss of non- 
myelinating functions of OLs, such as metabolic support, neurotransmitter homeostasis or clustering of voltage-gated ion channels ${ }^{39-42}$. Alternatively, the 3-week interim between tamoxifen administration and presentation of the complex wheel in which basal myelin formation is inhibited may affect learning performance independently of experience-induced OL production. In our study, we presented evidence against the latter possibility because we observed fear-learning deficits in Myrf icKO animals that were given tamoxifen both immediately before and after fear conditioning.

Remote memory consolidation, or systems consolidation, involves a reorganization of memory networks, over the course of several weeks or longer, such that retrieval of a memory transitions from dependence on the hippocampus to distributed cortical ensembles over time ${ }^{28,29}$. However, the synaptic, cellular and circuit-level mechanisms that underlie this process are not well understood. On the basis of lesioning studies and immediate early gene expression and functional optogenetics experiments, the mPFC is thought to be particularly important for remote memory $24,25,43,44$. We affirm this in our experiments by recording from a population of neurons over the course of a month, as a memory transitions from recent to remote, and demonstrating that the population response of the mPFC to identical context cues evolves over time, which, to our knowledge, has not been achievable with traditional electrophysiological recording methods. These findings are consistent with miniaturized microscopy imaging of the mPFC during fear conditioning, which indicates that a small subset of shock-responsive cells elevate their firing rates 15 days postconditioning when an animal is placed in the conditioned context ${ }^{25}$. Remarkably, this temporal evolution is not observed in the absence of new myelin formation, which, in conjunction with deficits in freezing behavior and immediate early gene expression specifically during remote recall, suggests that systems consolidation requires active myelination.

We observed fear-learning-induced changes in myelin content specifically in the mPFC but network-wide changes in immediate early gene expression, which is consistent with previous characterizations demonstrating that manipulating activity in a single node can have widespread effects on fear memory circuits ${ }^{27}$. Although the $\mathrm{mPFC}$ is dispensable for recent memory, input from the entorhinal cortex and BLA to the mPFC at the time of conditioning is required for the initial establishment of neuronal ensembles that subsequently mature to mediate remote memory retrieval ${ }^{25}$. Here, we describe an analogous situation in which OPC proliferation in the $\mathrm{mPFC}$ is elicited immediately at the time of conditioning, but the ensuing maturation of myelinating OLs occurs over a prolonged period that is similar in length to the approximate timeline reported for systems consolidation. Notably, we detected an increase in post-training proliferation of OPCs in the BLA that did not translate into an increase in myelinating OLs several weeks later, which suggests that these OPCs may have differentiated but failed to mature. This is in accordance with longitudinal two- photon imaging of OPC dynamics ${ }^{3}$ and suggests that although memory acquisition is important to initiate this sequence of events, it is perhaps the stable integration of maturing OLs during the consolidation period that is the important regulatory point in experience-dependent myelination. 
The mechanistic details of how new myelin formation acts to support remote memory is a salient question and requires a thorough understanding of how neuronal ensembles act in concert to achieve memory consolidation and retrieval. Although this remains an active area of research, the following points appear to be true: (1) systems consolidation involves substantial interregional reorganization of neuronal networks in the form of neuronal engram ensembles $24,25,28,43,44$; (2) active interregional communication between brain regions separated in space, arbitrated by the myelinated axons of projection neurons, is required for both successful memory consolidation and retrieval $24,25,27,45-48$; (3) this interregional communication seems to be characterized by a high degree of temporal precision, particularly in the form of synchronized oscillatory activity ${ }^{46,47}$. Thus, there are several conceivable ways through which new myelin formation can interact with or complement neural circuits to support systems consolidation and remote memory retrieval. Myelination could be strengthening specific projections in a manner akin to Hebbian plasticity, facilitating communication between spatially distributed neuronal ensembles, and/or tuning axonal conduction velocities such that synchronized oscillations or other computationally relevant firing patterns can be achieved ${ }^{20,48}$. A recent study reported episodic memory deficits and disruptions in ripple-spindle coupling between the MPFC and dHPC several days after tamoxifen administration in Myrf icKO animals ${ }^{49}$. While the timing of these observations is much more rapid than the timeline required for new, compact myelin formation that we have described here, ripple-spindle coupling represents a fascinating example of a memory consolidation mechanism that requires temporally precise interregional coordination and active oligodendrogenesis ${ }^{47,49}$. Collectively, these results indicate that experience-dependent OL production may affect circuit function through multiple mechanisms and across different time scales. Distinguishing between and elaborating on these many potential scenarios represents a major technical challenge and will likely require the development of tools for region-specific or projection-specific manipulations of new myelin formation in conjunction with recordings of neuronal ensembles across multiple brain regions in freely behaving animals.

In addition to establishing that remote fear memory requires new myelin formation, we demonstrated that induction of new myelin formation via clemastine enhances remote fear memory. As clemastine administration had no effect in Myrf icKO animals, this effect is likely dependent on new myelin formation. Notably, MRI examination of combat veterans with PTSD suggests that there is increased hippocampal myelin content compared with combat-exposed controls, which putatively implicates aberrant myelin formation in the pathophysiology of PTSD ${ }^{50}$. Although PTSD involves a constellation of symptoms, one of its prominent features is intense and overgeneralized fear responses ${ }^{21}$, which we have reproduced, to some degree, in mice chronically treated with clemastine. Indeed, a cardinal property of newly formed myelin is its stability, which may be desirable for retaining adaptive memories and learned skills, but detrimental if maladaptive fear responses are persistent and generalized to everyday situations ${ }^{1,2,18}$. Thus, investigating the role of new myelin formation in adaptive and maladaptive learning constitutes an important opportunity both for understanding, diagnosing and potentially treating PTSD and other neuropsychiatric illnesses, and for elucidating fundamental mechanisms of learning and memory. 


\section{online content}

Any methods, additional references, Nature Research reporting summaries, source data, extended data, supplementary information, acknowledgements, peer review information; details of author contributions and competing interests; and statements of data and code availability are available at https://doi.org/10.1038/s41593-019-0582-1.

\section{Methods}

Mice.

MyrfoxP/loxP (The Jackson Laboratory (JAX), 010607) mice were crossed to the NG2CreERT(JAX, 008538) line to generate MyrfoxP/loxP; NG2-CreERT(Myrf icKO) mice. These lines were maintained through crosses of $M y r f^{\text {foxP/loxP }} ; N G 2 C r e E R T^{+}$and $M y r f^{\text {foxP/loxP }}$;NG2CreERT $T^{+}$breeders, generating $M y r f^{\text {foxP/loxP }} ; N G 2 C r e E R T^{+}$and Myrf $^{\text {doxP/loxP }}$;NG2CreERT littermates on a mixed 129; CBA/J; C57BL/6J background. To validate the inhibition of new myelin formation following $M y r f$ deletion, triple transgenic Myrf $^{\text {oxP/loxP }}$;tau-mGFPloxP/loxP (JAX, 21162);NG2CreERT ${ }^{+}$and $\mathrm{Myr}^{\text {doxPl+ }}$;tau$m G P^{l o x P / l o x P} ; N G 2 C r e E R T^{+}$male and female littermates were generated through crosses of MyrfoxP/loxP,tau-mGFPloxP/loxP,NG2CreERT ${ }^{+}$and $M y r f^{\text {foxP/+ }}$;tau-

$m G F P^{l o x} / / 10 x P ; N G 2 C r e E R T^{-}$breeders. Cre-mediated recombination was induced via daily intraperitoneal injections of $20 \mathrm{mg} \mathrm{ml}^{-1}$ tamoxifen (Sigma-Aldrich) dissolved in 10\% ethanol/90\% peanut oil for five consecutive days, at a dose of $80 \mathrm{mg}$ per $\mathrm{kg}$. Mice were rested for either 3 weeks or 5 days before beginning subsequent behavioral experiments. The tau-mGFPloxP/loxP;NG2CreERT ${ }^{+}$mice on a C57BL/6J background were maintained through crosses of tau-mGFP $P^{\text {loxP/loxP }}$;NG2CreERT $T^{+}$and tau-mGFPloxP/loxP;NG2CreERT ${ }^{-}$breeders.

All transgenic mice used in this study were littermates aged 8-10 weeks at the start of experiments. Males and females were used in approximately equal numbers and agematched littermates were randomly assigned to cohorts. Genotyping was performed as recommended on the JAX datasheet for each mouse line via standard PCR of tail-derived genomic DNA.

Clemastine experiments in Figs. 7 and 8 and EdU experiments in Figs. 1 and 2 were conducted with 8-week-old male C57BL/6J mice (JAX, 000664). Clemastine fumarate (AvaChem Scientific) was prepared as a $1 \mathrm{mg} \mathrm{ml}^{-1}$ solution in $10 \%$ dimethylsulfoxide (DMSO; Sigma)/PBS and administered via daily intraperitoneal injections at a dose of 10 $\mathrm{mg}$ per $\mathrm{kg}$, alternating injection sites each day. Vehicle controls were injected in an identical manner with 10\% DMSO/PBS. EdU (Carbosynth) was prepared as a $10 \mathrm{mg} \mathrm{ml}^{-1}$ solution in PBS and administered via intraperitoneal injections at a dose of $80 \mathrm{mg}$ per $\mathrm{kg}$.

All animals were group-housed with one to four same-sex cagemates in standard rodent cages within a pathogen-free barrier facility on a 12-h light-dark cycle, with behavioral testing and recordings performed during the light phase. Food and water were available ad libitum and cages were changed twice a week by veterinary technicians. All procedures were preapproved by and conducted in accordance with the US NIH Guide for the Care and Use of Laboratory Animals and the Institutional Animal Care and Use Committees at the University of California, San Francisco. 


\section{Behavior.}

Conditioned fear was elicited by administering three mild foot shocks $(0.75 \mathrm{~mA})$, spaced 30 $\mathrm{s}$ apart, following a 5-min exposure to an array of contextual cues (conditioning chamber, chamber lights, white noise and scent of anise extract). Retrieval of the fear memory was assessed by reexposure of the animal to the conditioning context in the absence of shock, and freezing (the cessation of all movement outside of respiration) was interpreted as expression of fear memory. Video recordings were acquired and scored automatically in FreezeFrame (Actimetrics). Mice were habituated to transport and holding in a separate room for at least $1 \mathrm{~h}$ before all conditioning or retrieval sessions. For assessment of Fos induction following fear memory retrieval, habituation was extended to $4 \mathrm{~h}$ and all animals were perfused exactly $90 \mathrm{~min}$ after the start of the retrieval session. For assessment of generalization, freezing was measured in a context similar to the conditioning context, but with the following variations: chamber lights and fan were turned off, scent of anise extract was swapped for lemon extract and a plastic divider was inserted to make the chamber walls circular and opaque. Freezing in the similar context was tested $2 \mathrm{~h}$ following retrieval testing in the original conditioning context, and animals were rested in a holding room between sessions. For the immediate shock condition, one $0.75-\mathrm{mA}$ foot shock was administered $30 \mathrm{~s}$ after context exposure, followed by immediate removal of the animal from the chamber.

Anxiety-like behaviors were assessed via the OFT and EPM. Video recordings were acquired in Ethovision (Noldus) and the time spent in the periphery of the OFT and in the closed arms of the EPM were scored through automated object detection and interpreted as anxiety-like behavior. Freezing and anxiety-like behaviors were both scored via an automated, unbiased process and thus were not explicitly performed in a blinded manner.

\section{Fiber photometry.}

Recombinant AAV (AAV1.hSyn.GCaMP6f.WPRE.SV40 (ref. ${ }^{51}$ ), Addgene) was stereotactically injected unilaterally into the right $\mathrm{mPFC}(+1.7 \mathrm{~mm}$ anterior-posterior, +0.35 $\mathrm{mm}$ medial-lateral, $-2.75 / 2.5 / 2.25 / 2.0 \mathrm{~mm}$ dorsal-ventral, $150 \mathrm{nl}$ per injection site) of male

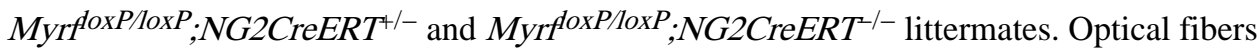
(400 $\mu \mathrm{m}$ in diameter and $2.2 \mathrm{~mm}$ in length) encased in stainless steel ferrules (Doric) were implanted directly above the injection site $(-1.9 \mathrm{~mm}$ dorsal-ventral) within the same surgery. After recovery from surgery over several days, mice were administered a 5-day tamoxifen regimen. Three weeks following surgery, animals were habituated to the patch cord (Doric) for $30 \mathrm{~min}$ for three consecutive days and then subjected to contextual fear conditioning as previously described. Fiber photometry recordings of mPFC calcium dynamics were acquired during each conditioning or retrieval session using the Synapse software suite (Tucker-Davis Technologies) and a hardware setup consisting of an RZ5P fiber photometry processor (Tucker-Davis Technologies), 405-nm and 465-nm light-emitting diode (LED) excitation (Doric), a six-port fluorescence mini cube (Doric) and photoreceiver module (Doric). LED excitation at $465 \mathrm{~nm}$ and $405 \mathrm{~nm}$ was sinusoidally modulated at 210 $\mathrm{Hz}$ and $330 \mathrm{~Hz}$, respectively. Patch cord autofluorescence was bleached overnight before every recording session via continuous LED illumination. A 5-min baseline recording in the home cage was acquired immediately before each conditioning or retrieval session. All animals were perfused for post hoc histology following their final recording session to verify 
viral expression and implant placement. One animal was excluded from the fiber photometry analysis due to incorrect fiber placement.

\section{Immunohistochemistry.}

Animals were perfused with PBS followed by $4 \%$ (w/v) paraformaldehyde (PFA) in PBS. Intact brains were extracted and post-fixedin $4 \%$ PFA overnight at $4{ }^{\circ} \mathrm{C}$. The tissue was then cryoprotected in $30 \%(\mathrm{w} / \mathrm{v})$ sucrose (Sigma) in PBS, sectioned at $30 \mu \mathrm{m}$ on a sliding microtome (Leica) and stored as floating sections in PBS containing $0.02 \%$ sodium azide. For immunohistological analysis, brain sections were blocked in $10 \%$ goat or donkey serum in PBS containing $0.1 \%(\mathrm{v} / \mathrm{v})$ Triton $\mathrm{X}-100$ for $1-2 \mathrm{~h}$ at room temperature. Primary antibodies were diluted in blocking solution and incubated overnight at $4{ }^{\circ} \mathrm{C}$, and secondary antibodies were diluted in PBS and incubated for $2 \mathrm{~h}$ at room temperature. The primary antibodies used in this study and their working concentrations are as follows: rabbit monoclonal anti-GFP (ThermoFisher, G10362; 1:1,000); chicken polyclonal anti-GFP (Aves Labs, CGFP-1020; 1:1,000); rabbit polyclonal anti-Olig2 (Millipore, AB9610; 1:1,000); rabbit polyclonal anti-ASPA (Sigma-Aldrich, ABN1698; 1:1,000); rat monoclonal anti-MBP (Serotec, MCA409S; 1:200); goat polyclonal anti-Fos (Santa Cruz Antibodies, sc-52-G; 1:500); and rabbit polyclonal anti-Iba1 (Wako, 019-19741; 1:1,000).

EdU incorporation by proliferating cells was detected via copper-catalyzed click chemistry with a picolyl azide conjugated to a 488 Alexa Fluor dye (Click Chemistry Tools). For colabeling with immunohistochemical staining, free-floating brain sections were incubated with a reaction mix consisting of 488 picolyl azide $(5 \mu \mathrm{M}), \mathrm{CuSO}_{4}$ (Sigma-Aldrich, $4 \mathrm{mM}$ ) and sodium ascorbate (Acros, $100 \mathrm{mM}$ ) in PBS for $30 \mathrm{~min}$ at room temperature between the blocking and primary antibody incubation steps.

\section{Electron microscopy.}

Eight-week-old male C57BL/6J mice were subjected to contextual fear conditioning and perfused 30 days post-conditioning with $2 \%$ (w/v) PFA/1.25\% (w/v) glutaraldehyde in 0.1 M sodium cacodylate buffer. Intact brains were extracted and post-fixed in 2\% PFA/1.25\% glutaraldehyde overnight at $4{ }^{\circ} \mathrm{C}$. The brains were then cryoprotected in $30 \%(\mathrm{w} / \mathrm{v})$ sucrose (Sigma) in PBS and sectioned coronally at $500 \mu \mathrm{m}$ on a sliding microtome (Leica). Sections containing the $\mathrm{mPFC}$ were then further cut by hand approximately along the dashed lines represented in Extended Data Fig. 1k and sent to the University of California, San Francisco Pathology Electron Microscopy Core facility for further processing and transmission electron microscopy imaging. Briefly, tissue was post-fixed in $2 \%$ osmium tetroxide in 0.1 M sodium cacodylate buffer, stained with $2 \%$ aqueous uranyl acetate, dehydrated in ethanol, cleared in propyline oxide and embedded in Eponate 12 (Ted Pella). Ultrathin sections were then collected, beginning from the medial margin, to sample the superficial cortical layers. The sections were subsequently stained with uranyl acetate and Reynold's lead and examined using a Tecnai 10 transmission electron microscope (Philips).

The presence of myelinated axons is highly variable in gray matter; therefore, to emphasize the regions of the mPFC with substantial myelin content, we subsampled the three fields of view, per animal, with the highest density of myelinated axons. The approximate region 
analyzed is represented in Extended Data Fig. 1k (green rectangle). This quantification was performed by an observer blinded to the experimental conditions.

\section{Fiber photometry analysis.}

Freezing behavior during fiber photometry experiments was scored manually in a blinded manner via The Observer (Noldus), as automated scoring was incompatible with the movement of the patch cord. Photometry signals were manually aligned to freezing behavior via a light pulse that was output by FreezeFrame and detected by an internally aligned video recording. The change in fluorescence intensity $(\Delta F F)$ was calculated by subtracting the linear fit of the isobestic signal to the raw GCaMP signal from the raw GCaMP signal and then dividing the subsequent result by the linear fit of the isobestic signal to the raw GCaMP signal; that is, $\left(465_{\text {raw }}-405_{\text {fitted }}\right) /\left(405_{\text {fitted }}\right)$. The $z$-scored $\Delta F / F$ values were then computed using the mean and standard deviation of the entire recording session. Peri-event analysis was centered on the transitions from bouts of mobility to bouts of freezing that each lasted at least $2 \mathrm{~s}$. The $\Delta F / F$ values were extracted from 2-s pre-transition and post-transition (referred to as pre and post), and the pre-mean and post-mean are the mean $z$-scored $\Delta F F$ values within the 2-s pre-bout and post-bout transition. Peri-event analyses for shock responses were similarly computed, comparing the mean $\Delta F F$ within the $5 \mathrm{~s}$ pre-shock and postshock.

\section{Histological quantification.}

All images were acquired using a CSU-W1 spinning disk confocal microscope (Nikon Imaging Center, UCSF). For all mPFC quantifications, nonoverlapping fields centered at the border between the IL and PL cortices were counted on each hemisection (Extended Data Fig. 1k). For the BLA and dHPC, the entire structure, per hemisection, was quantified as one field. Two fields were manually quantified per section using the multipoint tool in ImageJ, using two to three averaged sections per mouse. Absolute cell counts were normalized to the quantified area and reported as cellular density $\left(\right.$ cells $\left.\mathrm{mm}^{-2}\right)$. MBP fluorescence intensity was quantified by averaging the mean gray value (using the Measure function in Fiji ${ }^{52}$ ) from three nonoverlapping fields per brain region per section, with two to three averaged sections per mouse. All comparisons of MBP fluorescence intensity were conducted on sections stained and imaged within the same sessions, with identical exposure settings used during acquisition. For quantification of $\mathrm{GFP}^{+} \mathrm{MBP}^{+}$myelinating OLs, either cell bodies or nonoverlapping $\mathrm{GFP}^{+} \mathrm{MBP}^{+}$arbors were counted as individual cells, with occasional ambiguous cases conservatively quantified as a single cell. All image quantifications were conducted in a blinded manner.

\section{Statistical analysis.}

No statistical methods were used to predetermine samplesize a priori, but the sample sizes used were similar to those reported in previous studies ${ }^{15,17}$. Two-way analysis of variance (ANOVA) was used to probe for significant interactions of genotype with freezing behavior across contextual fear conditioning experiments, with Sidak's post hoc tests to make single comparisons between genotypes at specific time points. Single comparisons of histological quantifications and fiber photometry parameters were performed via two-tailed Student's $t$ tests; paired two-tailed $t$-tests were used for within animal comparisons and unpaired $t$-tests 
were used for comparisons between genotypes or treatment conditions. Comparisons of histological quantifications or freezing behavior between three or more groups or time points were performed using one-way ANOVA with Sidak's post hoc tests. Prism 6 (GraphPad) and Matlab (Mathworks) were used for all statistical analyses. Data distribution was assumed to be normal, but this was not formally tested. For all dot plots, data are presented as the mean \pm s.e.m. For all box-and-whisker plots, the center represents the median, the boxes represent the interquartile range and the whiskers represent the 10th and 90th percentiles. Sample sizes for graphs depicting pooled data and specific statistical tests used are indicated in the figure legends, with symbols indicating the following $P$ value ranges: $P>$ $0.05, * P \leq 0.05, * * P \leq 0.01, * * * P \leq 0.001$ and $* * * * P \leq 0.0001$; n.s. indicates not significant.

\section{Reporting Summary.}

Further information on research design is available in the Nature Research Reporting Summary linked to this article.

\section{Data availability}

The data that support the findings of this study are available from the corresponding authors upon reasonable request.

\section{Code availability}

Custom Matlab software written for extraction, analyses and visualization of photometry signals is available as Supplementary Software 1-3 and online at https://github.com/sp808/ Fiber-photometry.

\section{Extended Data}




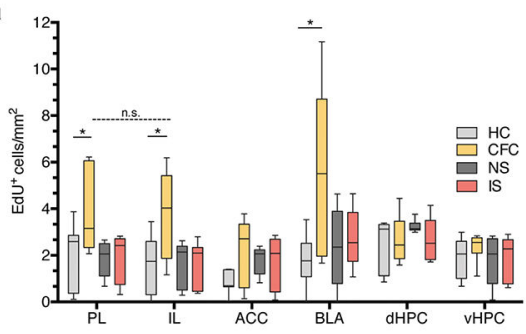

c
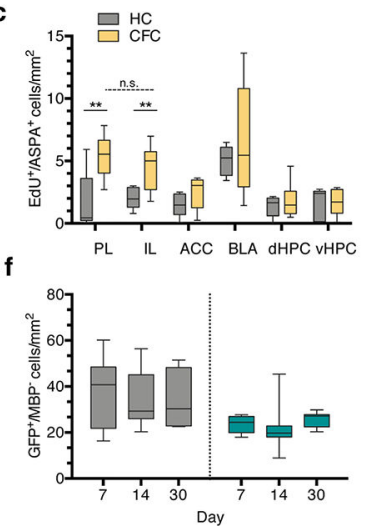

d b
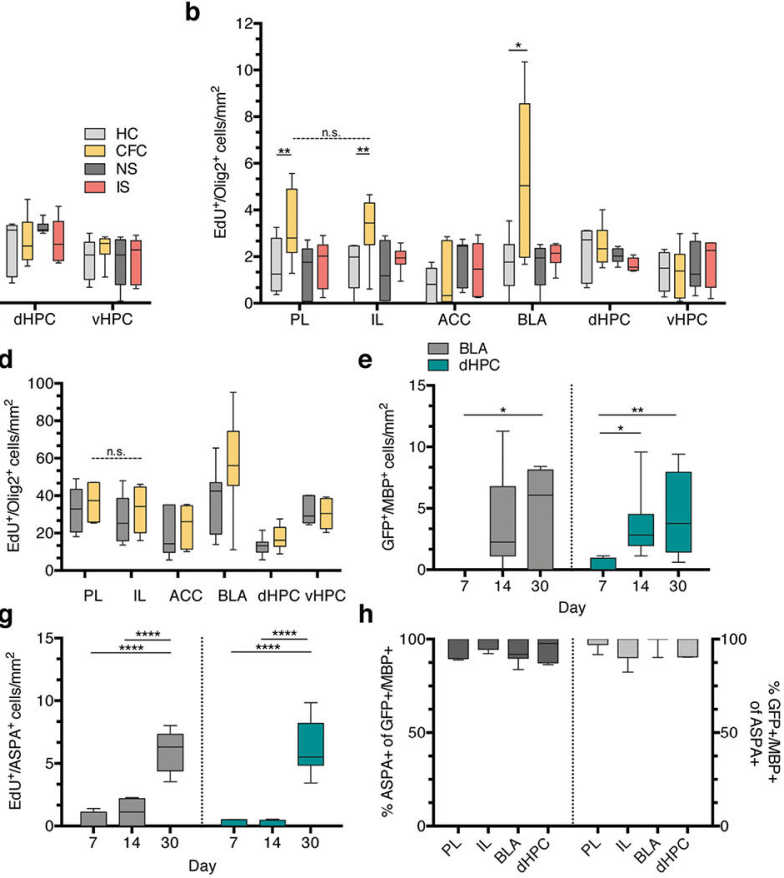
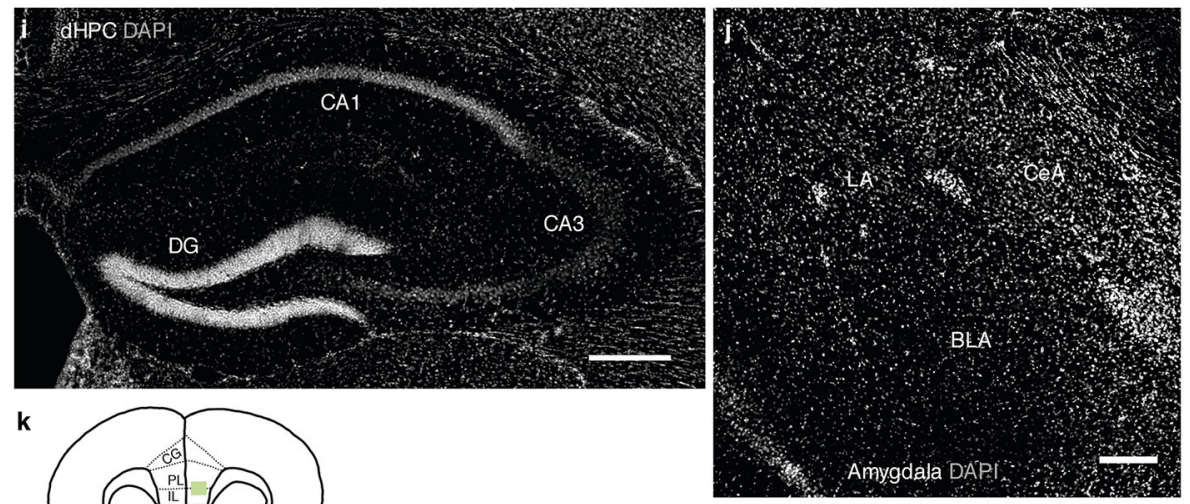

k

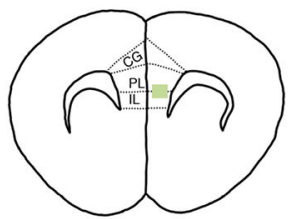

Extended Data Fig. 1 |. Proliferation, differentiation, and maturation of oPCs following fear learning.

a, EdU ${ }^{+}$cell density of home cage (HC), fear conditioned (CFC), no shock (NS), and immediate shock (IS) animals 24 hours post-conditioning; unpaired two-tailed t-tests comparing HC vs. CFC for PL (difference: $2.060 \pm 0.8565,95 \%$ CI: 0.1942 to $3.927, \mathrm{t}_{12}=$ 2.406, $\mathrm{p}=0.0332$ ), IL (difference: $2.239 \pm 0.8566,95 \%$ CI: 0.3731 to $4.106, \mathrm{t}_{12}=2.614, \mathrm{p}=$ 0.0226 ), ACC (difference: $1.176 \pm 0.5811,95 \%$ CI: -0.09067 to $2.442, \mathrm{t}_{12}=2.406, \mathrm{p}=$ 0.0760 ), BLA (difference: $3.865 \pm 1.395,95 \%$ CI: 0.8506 to $6.880, \mathrm{t}_{12}=2.77, \mathrm{p}=0.0159$ ), dHPC (difference: $2.060 \pm 0.8565,95 \%$ CI: 0.1942 to $3.927, \mathrm{t}_{12}=2.406, \mathrm{p}=0.6694$ ), vHPC (difference: $0.2300 \pm 0.5265,95 \% \mathrm{CI}:-0.9075$ to $1.368, \mathrm{t}_{12}=0.4369, \mathrm{p}=0.3128$ ), paired two-tailed t-test comparing PL vs. IL for CFC animals (difference: $-0.1025 \pm 0.7635,95 \%$ CI: -1.971 to $\left.1.766, \mathrm{t}_{6}=0.1343, \mathrm{p}=0.8975\right)$. $\mathbf{b}, \mathrm{EdU}^{+} / \mathrm{Olig} 2^{+}$cell density at 24 hours postconditioning; unpaired two-tailed t-tests comparing HC vs. CFC for PL (difference: $1.801 \pm$ $0.7408,95 \%$ CI: 0.1867 to $3.415, \mathrm{t}_{12}=2.431, \mathrm{p}=0.0317$ ), IL (difference: $1.547 \pm 0.6297$, 
95\% CI: 0.1750 to $2.919, \mathrm{t}_{12}=2.457, \mathrm{p}=0.0302$ ), ACC (difference: $0.2161 \pm 0.5518,95 \%$ CI: -0.9863 to $1.418, \mathrm{t}_{12}=0.3916, \mathrm{p}=0.7022$ ), BLA (difference: $3.619 \pm 1.338,95 \%$ CI: 0.7295 to $6.509, \mathrm{t}_{12}=2.706, \mathrm{p}=0.018$ ), dHPC (difference: $0.2882 \pm 0.4868,95 \%$ CI: -0.7634 to $1.340, \mathrm{t}_{12}=0.592, \mathrm{p}=0.564$ ), vHPC (difference: $-0.008014 \pm 0.5297,95 \% \mathrm{CI}$ : -1.162 to $1.146, \mathrm{t}_{12}=0.01513, \mathrm{p}=0.9882$ ), paired two-tailed $\mathrm{t}$-test comparing PL vs. IL for CFC animals (difference: $-0.2322 \pm 0.4552,95 \%$ CI: -1.346 to $0.8817, \mathrm{t}_{12}=0.5102, \mathrm{p}=$ 0.6289). For (a-b), $\mathrm{n}=7$ mice (HC), 8 mice (CFC), 7 mice (NS), 8 mice (IS). c, EdU ${ }^{+} / \mathrm{ASPA}^{2}$ + density post-conditioning; unpaired two-tailed t-tests HC vs. CFC for PL (difference: $3.576 \pm 1.006,95 \%$ CI: 1.404 to $5.749, \mathrm{t}_{13}=3.556, \mathrm{p}=0.035$ ), IL (difference: $2.472 \pm$ $0.7097,95 \%$ CI: 0.9503 to $3.995, \mathrm{t}_{13}=0.5102, \mathrm{p}=0.037$ ), ACC (difference $0.9596 \pm$ $0.5516,95 \%$ CI: -0.2234 to $2.143, \mathrm{t}_{13}=0.5102, \mathrm{p}=0.1038$ ), BLA (difference: $1.520 \pm$ $2.071,95 \%$ CI: -3.165 to $6.204, \mathrm{t}_{13}=0.7339, \mathrm{p}=0.4817$ ), dHPC (difference: $0.3704 \pm$ $0.5950,95 \%$ CI: -0.9150 to $1.656, \mathrm{t}_{13}=0.6225, \mathrm{p}=0.5444$ ), vHPC (difference: $0.0536 \pm$ $0.6227,95 \%$ CI: -1.303 to $1.410, \mathrm{t}_{13}=0.086, \mathrm{p}=0.9328$ ), paired two-tailed t-test comparing PL vs. IL for CFC animals (difference: $-0.9171 \pm 0.7719,95 \%$ CI: -2.742 to $\left.0.9081, \mathrm{t}_{13}=1.188, \mathrm{p}=0.5466\right) . \mathbf{d}, \mathrm{EdU}^{+} / \mathrm{Olig} 2^{+}$density 30 days post-conditioning; unpaired two-tailed t-tests $\mathrm{HC}$ vs. CFC for PL (difference: $4.512 \pm 5.558,95 \% \mathrm{CI}:-7.599$ to $16.62, \mathrm{t}_{13}=0.8117, \mathrm{p}=0.4328$ ), IL (difference: $4.514 \pm 6.705,95 \% \mathrm{CI}:-10.10$ to $19.12, \mathrm{t}_{13}$ $=0.6731, \mathrm{p}=0.5136$ ), ACC (difference: $3.086 \pm 6.324,95 \%$ CI: -10.69 to $16.86, \mathrm{t}_{13}=$ $0.488, \mathrm{p}=0.6343$ ), BLA (difference: $19.00 \pm 11.32,95 \% \mathrm{CI}:-5.452$ to $43.45, \mathrm{t}_{13}=1.679, \mathrm{p}$ $=0.1171$ ), $\mathrm{dHPC}$ (difference: $4.325 \pm 2.933,95 \% \mathrm{CI}:-2.011$ to $10.66, \mathrm{t}_{13}=1.475, \mathrm{p}=$ 0.1641 ), vHPC (difference: $-0.8542 \pm 3.854,95 \%$ CI: -9.251 to $7.543, \mathrm{t}_{13}=0.2216, \mathrm{p}=$ 0.8283 ), paired two-tailed t-test comparing PL vs. IL for CFC animals (difference: $-4.630 \pm$ $6.858,95 \%$ CI: -21.41 to $\left.12.15, \mathrm{t}_{13}=0.6751, \mathrm{p}=0.5248\right)$. For $(\mathbf{c}-\mathbf{d}), \mathrm{n}=7$ mice $(\mathrm{HC})$ and 8 mice (CFC). Quantification of $\mathrm{GFP}^{+} / \mathrm{MBP}^{+}(\mathbf{e}), \mathrm{GFP}^{+} / \mathrm{MBP}^{-}(\mathbf{f})$, and $\mathrm{EdU}^{+} / \mathrm{ASPA}^{+}(\mathbf{g})$ cell density in the dHPC and BLA; $\mathrm{n}=6$ mice ( 7 days), 9 mice (14 days), and 7 mice ( 30 days). e-g One-way ANOVA with Sidak's post hoc tests comparing cell densities across days; (e) BLA: $\mathrm{F}_{2,19}=4.369, \mathrm{p}=0.0275,7$ vs. 14 day, (difference: $-3.760 \pm 1.672,95 \%$ CI: -7.818 to $0.2981, \mathrm{p}=0.0718$ ), 7 vs. 30 day (difference: $-5.041 \pm 1.765,95 \%$ CI: -9.324 to $-0.7572, \mathrm{p}=0.0201$ ), dHPC: $\mathrm{F}_{2,22}=5.397, \mathrm{p}=0.0124,7$ vs. 14 day, (difference: $-3.108 \pm$ $1.241,95 \%$ CI: -6.086 to $-0.1300, \mathrm{p}=0.0399$ ), 7 vs. 30 day (difference: $-4.124 \pm 1.303$, 95\% CI: -7.251 to $-0.9968, \mathrm{p}=0.009$ ); (f) BLA: $\mathrm{F}_{2,19}=0.07572, \mathrm{p}=0.9274,7$ vs. 14 day, (difference: $2.354 \pm 6.908,95 \%$ CI: -14.41 to $19.12, p=0.9308$ ), 7 vs. 30 day (difference: $2.540 \pm 7.292,95 \%$ CI: -15.16 to $20.24, p=0.9279)$, dHPC: $F_{2,20}=0.4852, p=0.6226,7$ vs. 14 day, (difference: $1.629 \pm 3.759,95 \%$ CI: -7.456 to $10.71, \mathrm{p}=0.8907), 7$ vs. 30 day (difference: $-1.900 \pm 4.049,95 \%$ CI: -11.69 to $7.888, \mathrm{p}=0.8733$ ); (g) BLA: $\mathrm{F}_{2,18}=47.49$, p < 0.0001, 7 vs. 14 day, (difference: $-0.6155 \pm 0.6763,95 \%$ CI: -2.265 to $1.034, p=$ 0.6092 ), 7 vs. 30 day (difference: $-5.632 \pm 0.6565,95 \%$ CI: -7.233 to -4.031 , $<<0.0001$ ), dHPC: $F_{2,16}=46.74, p<0.0001,7$ vs. 14 day, (difference: $-0.03272 \pm 0.7020,95 \%$ CI: -1.764 to $1.699, \mathrm{p}=0.9987$ ), 7 vs. 30 day (difference: $-6.038 \pm 0.7285,95 \% \mathrm{CI}:-7.835$ to $-4.242, \mathrm{p}<0.0001$ ). $\mathbf{h}$, Proportion of $\mathrm{GFP}^{+} / \mathrm{MBP}^{+}$cells that are $\mathrm{ASPA}^{+}$(left bars) and the proportion of $\mathrm{ASPA}^{+} / \mathrm{GFP}^{+}$cells that are $\mathrm{MBP}^{+}$(right bars) at 30 days post-conditioning; $\mathrm{n}$ $=7$ mice. Representative DAPI (gray) images the sub-regional dHPC (i) and amygdalar (j) cytoarchitecture, scale bars: $250 \mu \mathrm{m}(\mathbf{k})$ Schematic of the sampled area within the mPFC (green rectangle); dashed lines demarcate the approximate region cut for electron 
microscopy. For box-and-whisker plots, the center, boxes, and whiskers represent the median, interquartile range, and the $10^{\text {th }}$ and $90^{\text {th }}$ percentiles, with asterisks indicating the following p-value ranges: $* \leq 0.05, * * \leq 0.01, * * * \leq 0.001, * * * * \leq 0.0001$. 

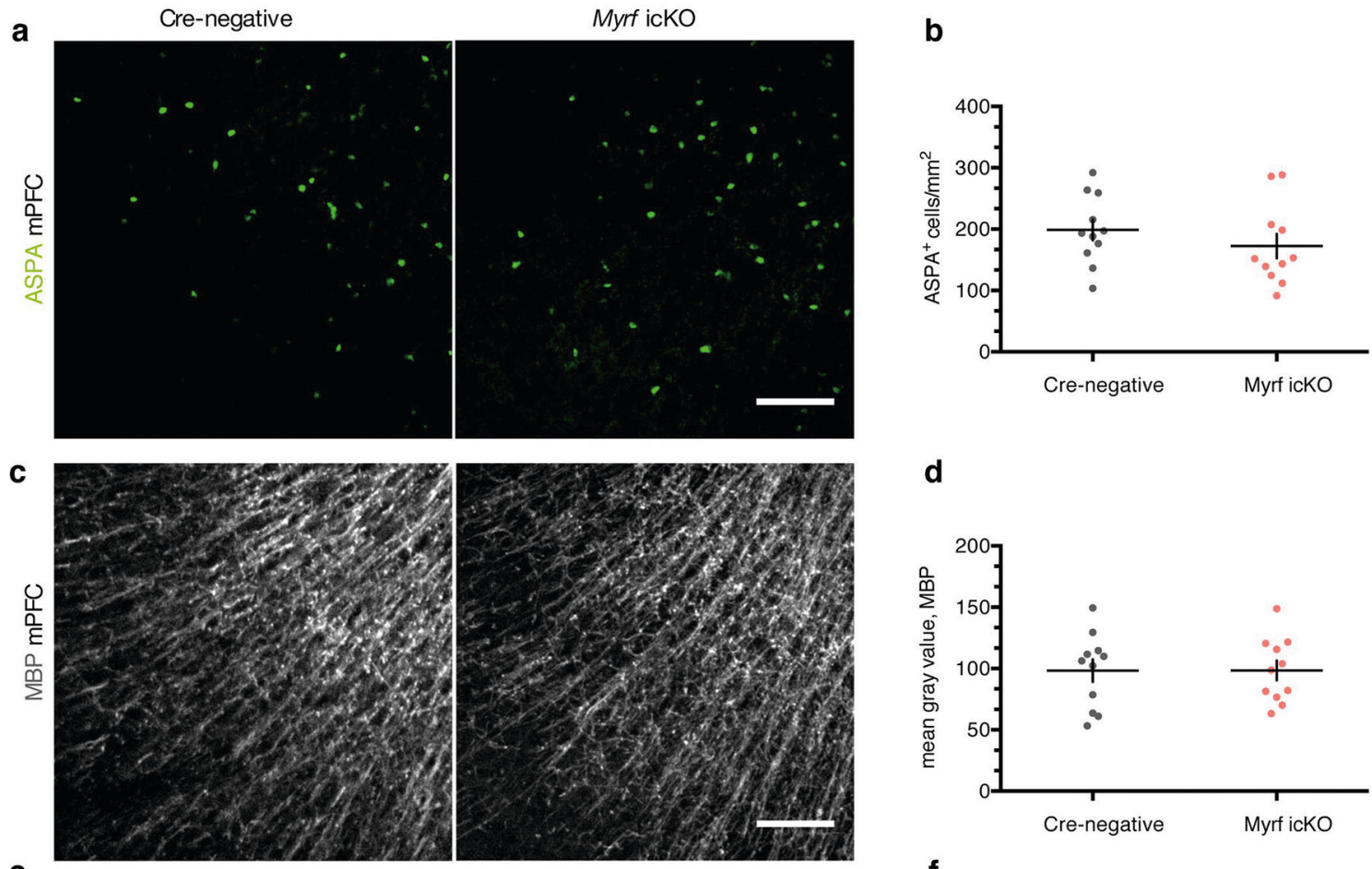

d
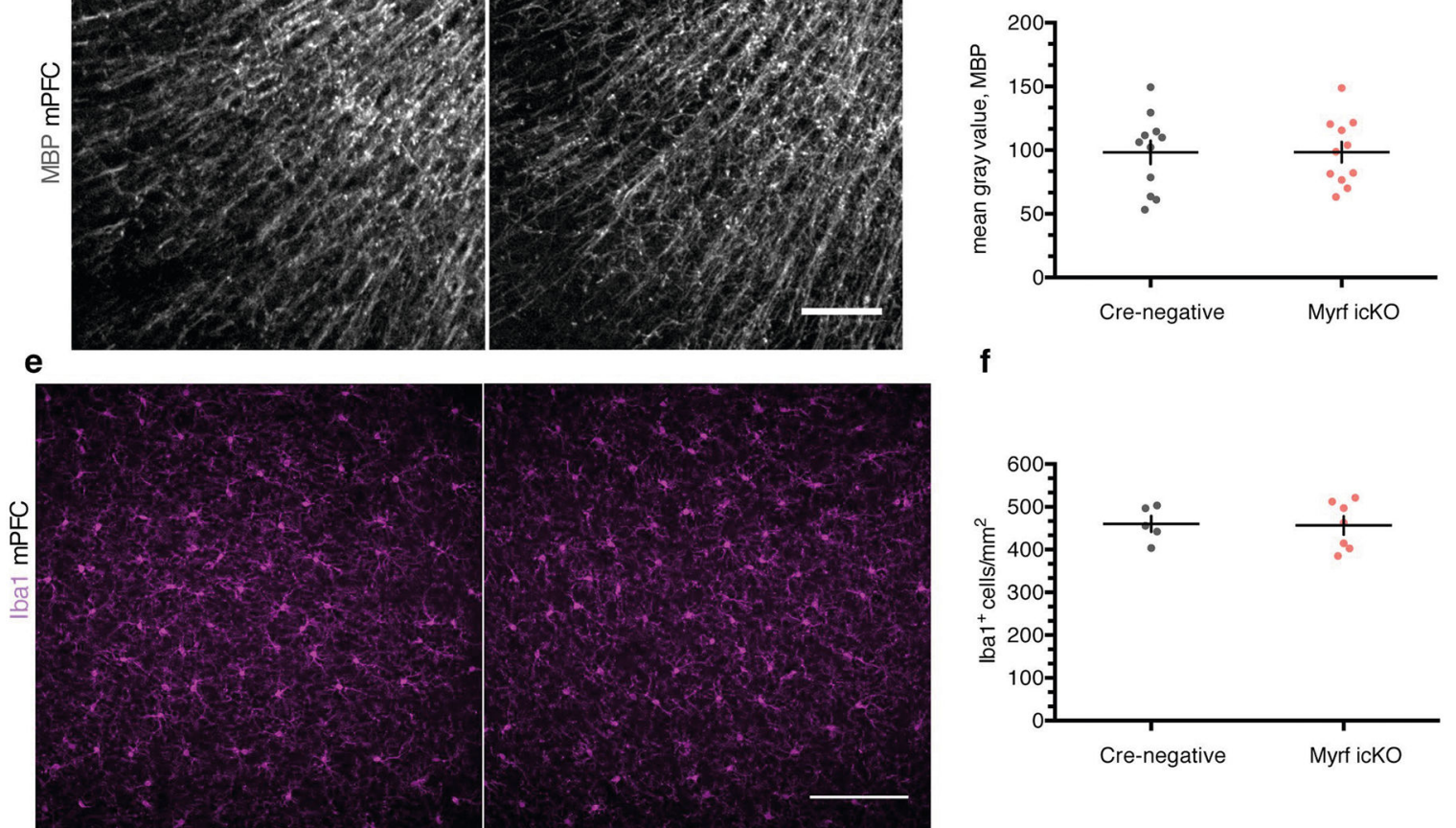

Extended Data Fig. 2 . overall myelination status and microglial density are unchanged in the absence of new myelin formation.

a, Representative images of ASPA (green) staining in the PFC of cre-negative and Myrf icKO mice 30 days post-conditioning, quantified in (b); unpaired two-tailed t-test, difference: $-26.50 \pm 26.20,95 \%$ CI: -81.15 to $28.15, \mathrm{t}_{20}=1.012, \mathrm{p}=0.3238, \mathrm{n}=11$ mice per genotype. c, Representative images of MBP (gray) staining in the PFC of cre-negative and Myrf icKO mice 30 days post-conditioning, quantified in (d); unpaired two-tailed t-test, difference: $0.1927 \pm 12.13,95 \%$ CI: -25.12 to $25.50, \mathrm{t}_{20}=2.406, \mathrm{p}=0.9875, \mathrm{n}=11$ mice per genotype. e, Representative images of Iba1 (magenta) staining in the PFC of cre-negative and Myrf icKO mice 30 days post-conditioning, quantified in (f); unpaired two-tailed t-test, difference: $-3.698 \pm 29.55,95 \%$ CI: -69.53 to $62.14, \mathrm{t}_{10}=0.1251, \mathrm{p}=0.9029, \mathrm{n}=6$ mice per genotype. Scale bars: $100 \mu \mathrm{m}(\mathbf{a}, \mathbf{c}, \mathbf{e})$. Data are presented as mean \pm SEM. 


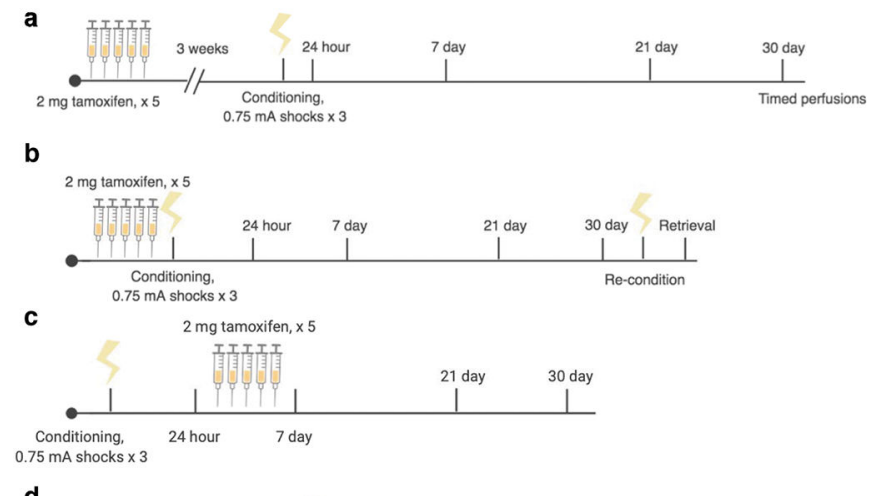

d

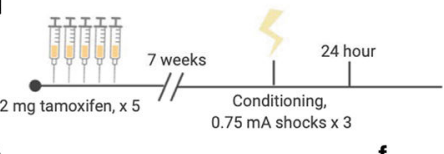

e

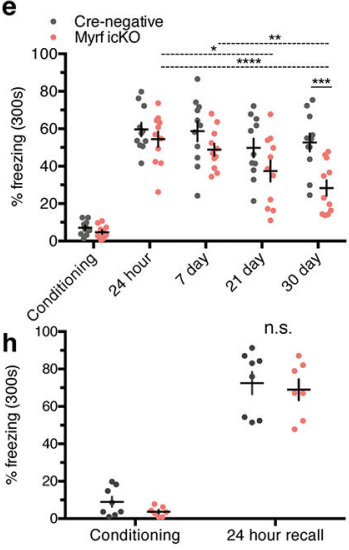

f
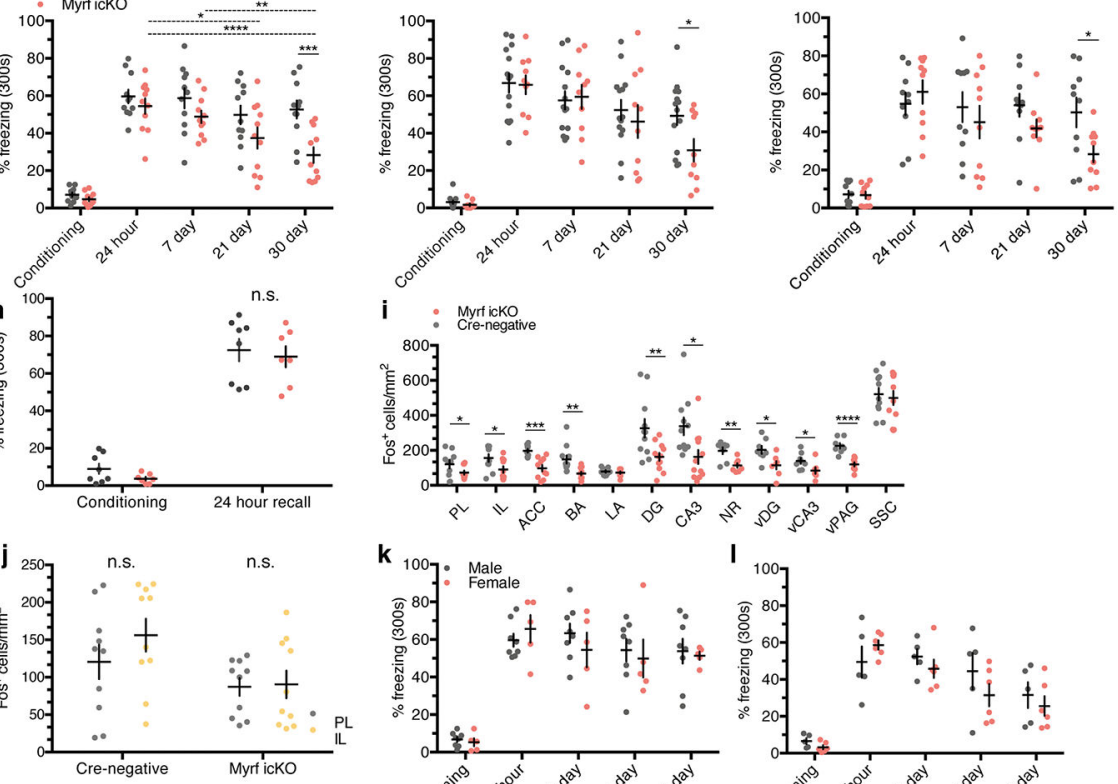

i : Myrricko
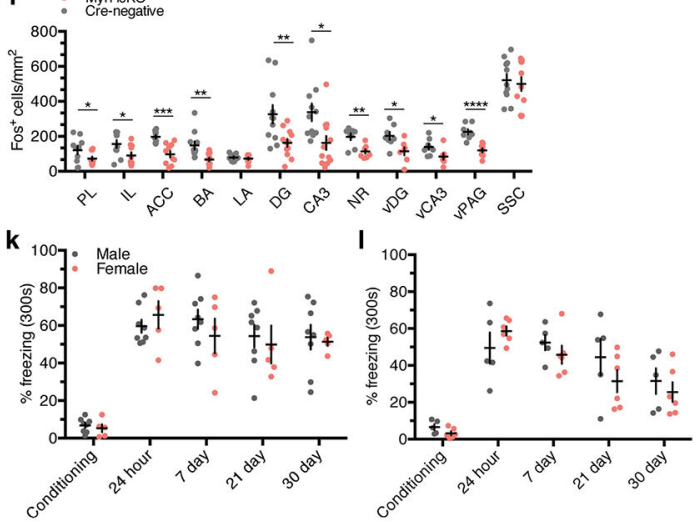

Extended Data Fig. 3 |. inhibition of new myelin formation impairs remote fear memory recall. a, Extended experimental timeline for cohort represented in Fig. 4a,e, quantified in (e); twoway ANOVA $\left(\mathrm{F}_{4,80}=3.437, \mathrm{p}=0.0121\right)$ with Sidak's post hoc tests comparing cre-negative vs. Myrf icKO during conditioning (difference: $-2.416 \pm 5.790,95 \% \mathrm{CI}:-17.58$ to $12.74, \mathrm{p}$ $=0.9965$ ), 24 hour (difference: $-5.169 \pm 5.790,95 \%$ CI: -20.33 to $9.991, p=0.9040$ ), 7 day (difference: $-9.901 \pm 5.790,95 \% \mathrm{CI}:-25.06$ to $5.260, \mathrm{p}=0.9040), 21$ day (difference: $-12.35 \pm 5.790,95 \% \mathrm{CI}:-27.51$ to $2.811, \mathrm{p}=0.3772$ ), and 30 day (difference: $-24.37 \pm$ $5.790,95 \% \mathrm{CI}:-39.53$ to $-9.209, \mathrm{p}=0.0003$ ), and comparing 7 day (difference: $5.685 \pm$ 5.685, 95\% CI: -6.004 to $17.37, \mathrm{p}=0.6277$ ), 21 day (difference: $17.05 \pm 17.05,95 \% \mathrm{CI}$ : 5.361 to $28.74, \mathrm{p}=0.0015$ ), and 30 day (difference: $26.18 \pm 26.18,95 \% \mathrm{CI}: 14.49$ to 37.87 , $\mathrm{p}<0.0001$ ) to 24 hour freezing within MyrficKO animals, $\mathrm{n}=11$ animals per genotype. $\mathbf{b}$, Extended experimental timeline for cohort represented in Fig. 4b,f,g, quantified in (f); twoway ANOVA $\left(\mathrm{F}_{4,80}=1.435, \mathrm{p}=0.2291\right)$ with Sidak's post hoc tests comparing cre-negative 
vs. Myrf icKO during conditioning (difference $1.467 \pm 7.398,95 \%$ CI: -13.19 to $16.13, \mathrm{p}=$ 0.8431 ), 24 hour (difference: $0.9199 \pm 7.398,95 \% \mathrm{CI}:-13.74$ to $15.58, \mathrm{p}=0.9013$ ), 7 day (difference: $-1.946 \pm 7.398,95 \% \mathrm{CI}:-16.61$ to $12.71, \mathrm{p}=0.7930$ ), 21 day (difference: 6.165 \pm 7.398 , $95 \%$ CI: -8.496 to $20.83, p=0.4064$ ), and 30 day (difference: $18.43 \pm 7.398,95 \%$ CI: 3.767 to $33.09, \mathrm{p}=0.0142), \mathrm{n}=14$ cre-negative and 10 Myrf icKO mice. $\mathbf{c}$,

Experimental timeline for cohort in which tamoxifen was administered after 24 hour recall, quantified in $(\mathbf{h})$; two-way ANOVA $\left(\mathrm{F}_{4,72}=1.724, \mathrm{p}=0.1541\right), \mathrm{n}=10$ animals per genotype. d, Experimental timeline for cohort in which tamoxifen was administered seven weeks prior to conditioning, quantified in (h); two-way ANOVA (genotype, $\mathrm{F}_{1,26}=0.03642$, $\mathrm{p}=0.8501)$ with Sidak's post hoc tests comparing cre-negative vs. Myrf icKO during conditioning (difference: $5.241 \pm 6.245,95 \% \mathrm{CI}$ : -9.579 to $20.06, \mathrm{p}=0.6507$ ) and 24 hour (difference: $3.556 \pm 6.245,95 \% \mathrm{CI}:-11.26$ to $18.37, \mathrm{p}=0.8185$ ), $\mathrm{n}=8$ cre-negative and 7 Myrf icKO mice. i, Expanded quantification of $\mathrm{Fos}^{+}$cell density across brain regions following 30-day retrieval sessions; unpaired two-tailed t-tests, PL (difference: $62.14 \pm$ 24.56, 95\% CI: 10.54 to $113.7, \mathrm{t}_{21}=72.53, \mathrm{p}=0.0209$ ), IL (difference: $65.70 \pm 28.64,95 \%$ CI: 5.532 to $125.9, \mathrm{t}_{21}=2.294, \mathrm{p}=0.034$ ), ACC (difference: $99.84 \pm 21.22,95 \%$ CI: 55.27 to $144.4, \mathrm{t}_{21}=4.706, \mathrm{p}=0.0002$ ), BA (difference: $80.55 \pm 24.87,95 \%$ CI: 28.66 to 132.4 , $\mathrm{t}_{21}=3.238, \mathrm{p}=0.0041$ ), LA (difference: $6.494 \pm 9.138,95 \% \mathrm{CI}:-12.70$ to $25.69, \mathrm{t}_{21}=$ 0.7107, $\mathrm{p}=0.4864$ ), DG (difference: $163.5 \pm 55.29,95 \% \mathrm{CI}: 48.50$ to $278.5, \mathrm{t}_{21}=2.957, \mathrm{p}=$ 0.0075), CA3 (difference: $175.4 \pm 63.83,95 \%$ CI: 42.70 to $308.2, \mathrm{t}_{21}=2.749, \mathrm{p}=0.012$ ), NR (difference: $84.08 \pm 22.50,95 \%$ CI: 35.83 to $132.3, \mathrm{t}_{21}=3.737, \mathrm{p}=0.0022$ ), $\mathrm{vDG}$ (difference: $88.20 \pm 32.23,95 \%$ CI: 18.58 to $157.8, \mathrm{t}_{21}=2.737, \mathrm{p}=0.017$ ), vCA3 (difference: $55.39 \pm 24.33,95 \%$ CI: 2.831 to $108.0, \mathrm{t}_{21}=7.361, \mathrm{p}=0.0404$ ), $\mathrm{vPAG}$ (difference: $105.9 \pm 18.71,95 \%$ CI: 66.04 to $145.8, \mathrm{t}_{21}=5.662, \mathrm{p}<0.0001$ ), SCC (difference: $21.86 \pm 53.48,95 \%$ CI: -89.35 to $133.1, \mathrm{t}_{21}=0.4088, \mathrm{p}=0.6868$ ), $\mathrm{n}=12$ crenegative and 11 Myrf icKO mice. $\mathbf{j}$, Within-genotype comparisons of $\mathrm{Fos}^{+}$cell density between the PL and IL; paired two-tailed t-tests, cre-negative (difference: $35.68 \pm 33.66$, 95\% CI: -40.47 to $111.8, \mathrm{t}_{9}=0.4088, \mathrm{p}=0.3168$ ), Myrf icKO (difference: $3.320 \pm 21.65$, $95 \%$ CI: -45.65 to $\left.52.29, \mathrm{t}_{9}=0.4088, \mathrm{p}=0.8815\right), \mathrm{n}=10$ mice per genotype. Withingenotype comparisons of freezing responses between males and females for animals represented in a-e for cre-negative (k) and Myrf icKO animals (l); two-way ANOVA for crenegative $\left(\mathrm{F}_{4,44}=0.6815, \mathrm{p}=0.6085\right)$ and MyrficKO animals $\left(\mathrm{F}_{4,44}=1.649, \mathrm{p}=0.1833\right), \mathrm{n}$ $=8$ males and 5 females for cre-negative mice, $\mathrm{n}=5$ males and 6 females for Myrf icKO mice. Data are presented as mean \pm SEM, with asterisks indicating the following $\mathrm{p}$-value ranges: $* \leq 0.05, * * \leq 0.01, * * * \leq 0.001, * * * * \leq 0.0001$. 
a

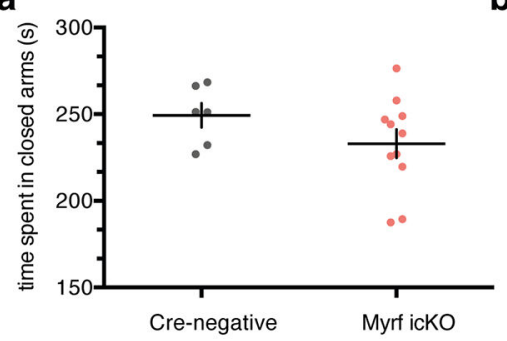

b

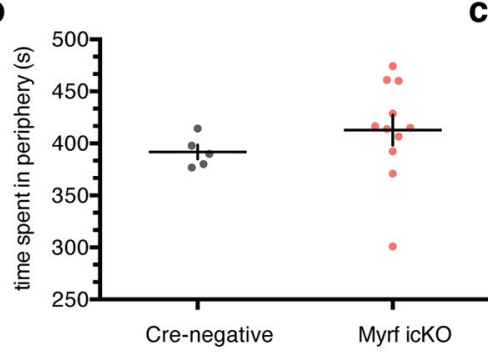

c

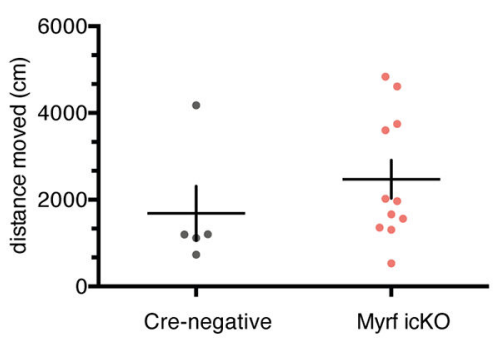

Cre-negative

d
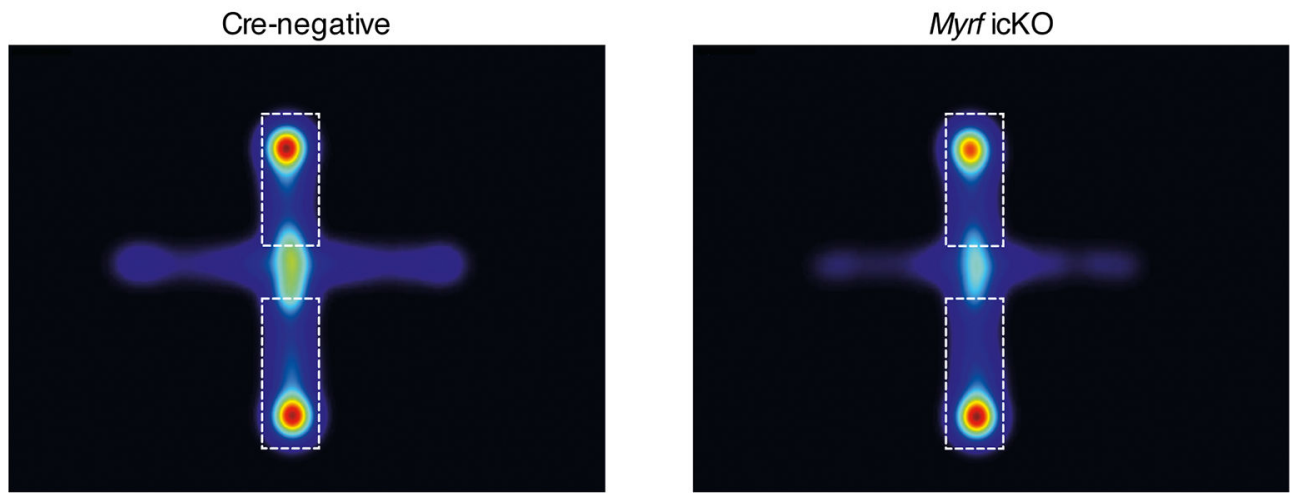

e
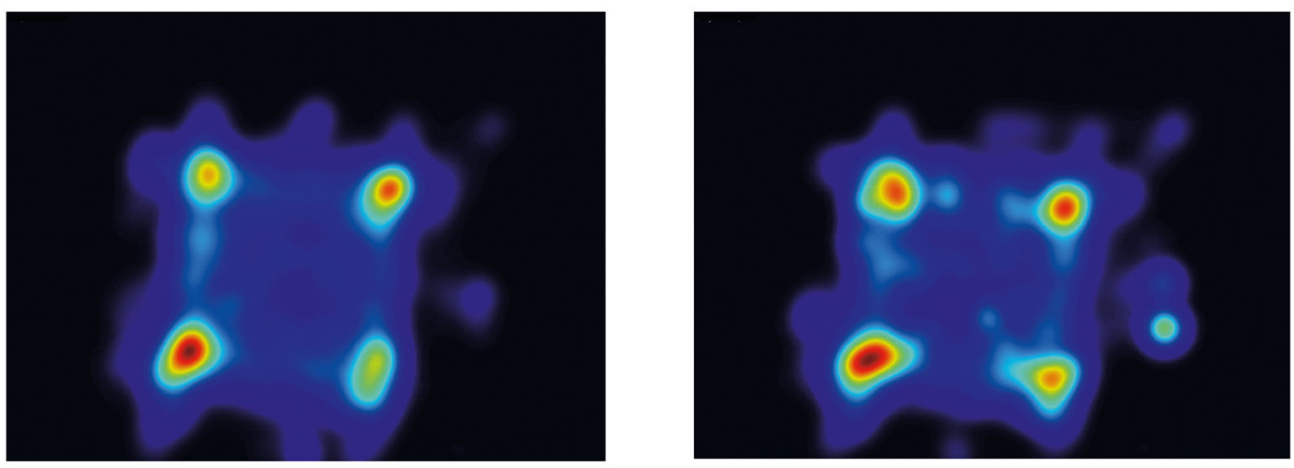

Extended Data Fig. 4 |. innate anxiety-like and locomotor behaviors are unchanged in the absence of new myelin formation.

Quantification of time spent in the closed arms of the elevated plus maze (EPM) (a) and the periphery of the open field test (OFT) (b); unpaired two-tailed t-test, EPM (difference:

$-16.38 \pm 12.29,95 \% \mathrm{CI}:-42.59$ to $9.818, \mathrm{t}_{15}=1.333, \mathrm{p}=0.2025$ ), OFT (difference: 20.94 $\pm 22.43,95 \%$ CI: -27.17 to $\left.69.05, \mathrm{t}_{15}=0.9334, \mathrm{p}=0.3664\right)$. (c) Quantification of total distance traveled in the open field test; unpaired two-tailed t-test (difference: $788.3 \pm 780.0$, $95 \%$ CI: -884.6 to $2461, \mathrm{t}_{15}=1.011, \mathrm{p}=0.3293$ ). Heatmaps representing relative frequency (red - frequent, blue - infrequent) of cre-negative and MyrficKO animals in the elevated plus maze (d) (dashed lines demarcate the closed arms), and open field test (e). Data are presented as mean \pm SEM. For a-c, $n=5$ cre-negative and 11 Myrf icKO mice. 
a

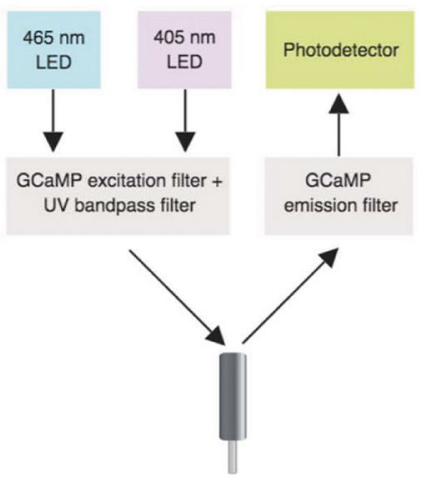

C

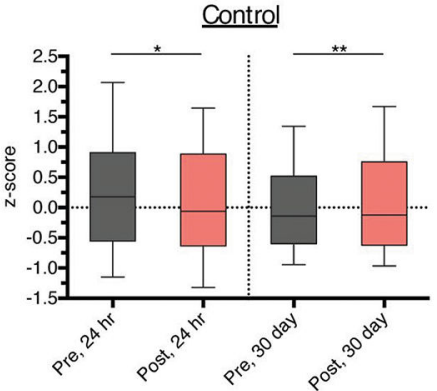

f

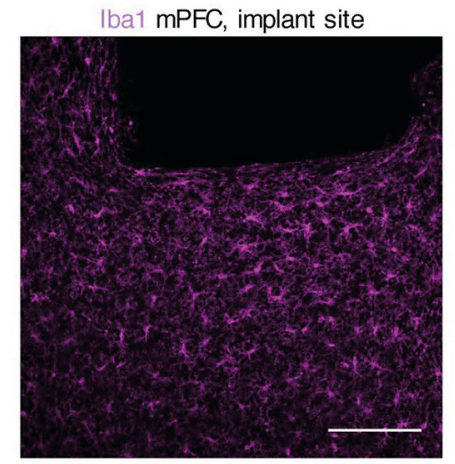

\section{b}

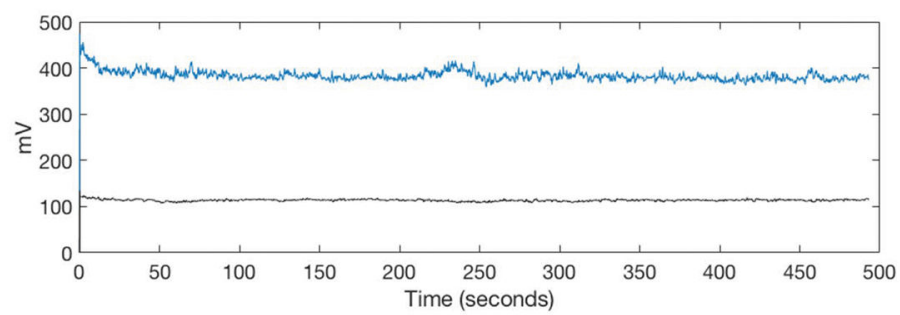

d

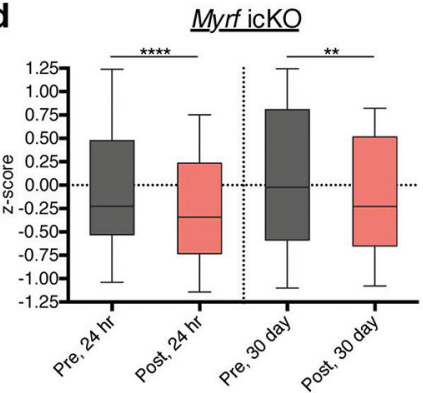

e

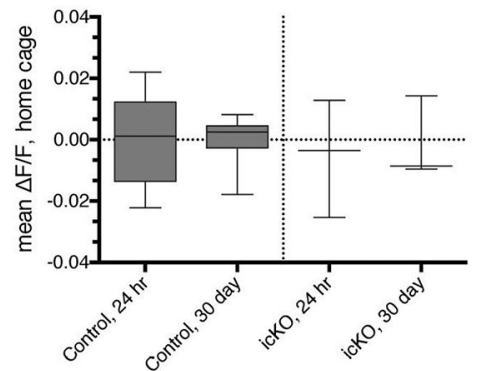

\section{g}

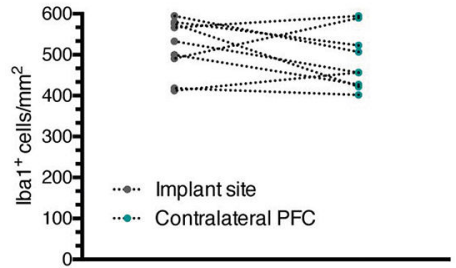

Extended Data Fig. $5 \mid$. Supplementary data for fiber photometry experiments.

$\mathbf{a}$, Schematic of fiber photometry recording setup. $\mathbf{b}$, Representative trace of a recorded retrieval session depicting the raw GCaMP (blue) and isobestic (black) signal, with no detectable motion artifact. Quantification of the z-scored mean $\Delta \mathrm{F} / \mathrm{F}$ during the two seconds pre- and post-bout transition at 24 hours and 30 days for control (c) and Myrf icKO (d) animals; $\mathrm{n}=10$ bouts across 7 mice (controls) and 7 mice (Myrf icKO); paired two-tailed ttests comparing z-scored signal intensity pre- and post-bout for control 24 hour (difference: $-0.2016 \pm 0.1564,95 \%$ CI: -0.5095 to $\left.0.1064, \mathrm{t}_{138}=1.289, \mathrm{p}=0.0202\right)$, control 30 day (difference $0.1051 \pm 0.1118,95 \% \mathrm{CI}:-0.1150$ to $0.3251, \mathrm{t}_{138}=0.9396, \mathrm{p}=0.055$ ), Myrf icKO 24 hour (difference: $-0.2216 \pm 0.1650,95 \%$ CI: -0.5494 to $0.1063, \mathrm{t}_{138}=1.343, \mathrm{p}<$ 0.0001 ), and Myrf icKO 30 day (difference: $-0.1837 \pm 0.1502$, 95\% CI: -0.4810 to 0.1136 , $\left.\mathrm{t}_{138}=1.223, \mathrm{p}=0.0032\right)$. $\mathrm{e}$, Quantification of the mean $\Delta \mathrm{F} / \mathrm{F}$ over a five-minute home cage recording taken just prior to the 24-hour and 30-day retrieval sessions; $\mathrm{n}=7$ (controls) and 7 mice (Myrf icKO); unpaired two-tailed t-tests, 24 hour (difference: $-0.0001284 \pm 0.006472$, 
95\% CI: -0.01423 to $0.01397, \mathrm{t}_{13}=0.01984, \mathrm{p}=0.6534$ ), 30 day (difference: $0.004054 \pm$ $0.01353,95 \%$ CI: -0.03350 to $0.04161, \mathrm{t}_{13}=0.9334, \mathrm{p}=0.8892$ ). $\mathbf{f}$, Representative image of Iba1 staining under the implant site, quantified in $(\mathbf{g})$; paired two-tailed t-test (difference: $-32.40 \pm 26.10,95 \%$ CI: -92.58 to $\left.27.78, \mathrm{t}_{16}=0.908, \mathrm{p}=0.2496\right), \mathrm{n}=9$ mice. Scale bar: $100 \mu \mathrm{m}$. For box-and-whisker plots, the center, boxes, and whiskers represent the median, interquartile range, and the $10^{\text {th }}$ and $90^{\text {th }}$ percentiles, with asterisks indicating the following p-value ranges: $* \leq 0.05, * * \leq 0.01, * * * * \leq 0.0001$. 
y.

to 21 days post-conditioning

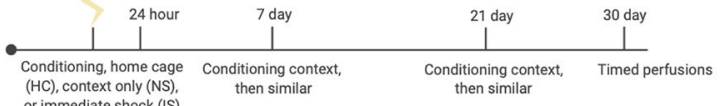

(2)

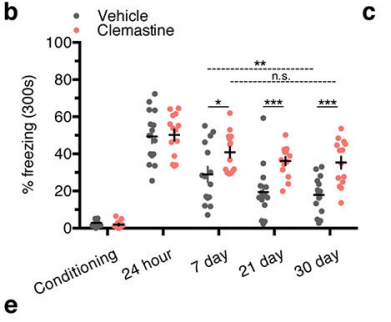

C

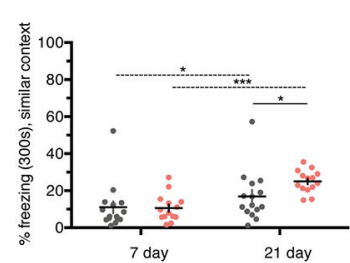

d
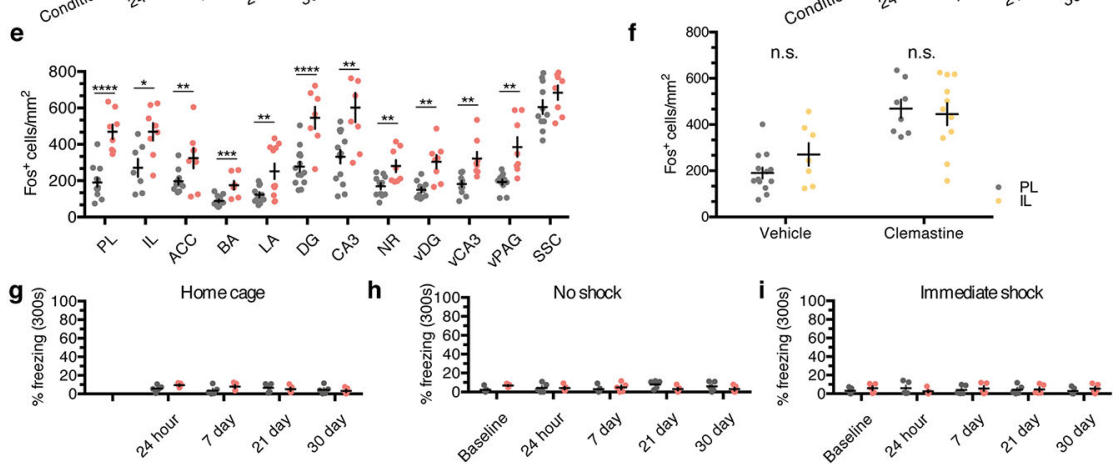

j

$\mathbf{k}$
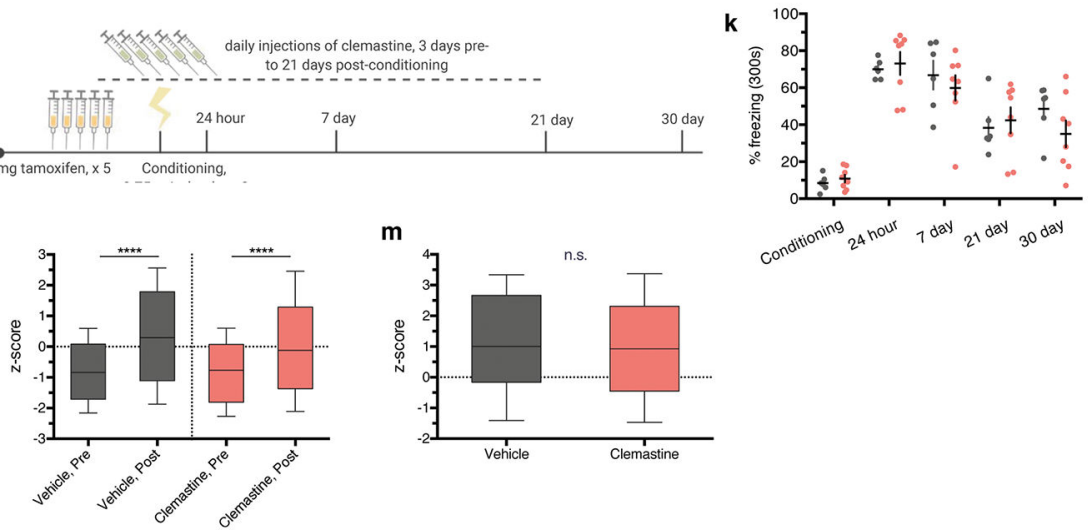

Extended Data Fig. 6 |. induction of new myelin formation preserves remote fear memory recall. a, Extended experimental timeline for cohort represented in Fig. 7a,e. b, Freezing responses in the conditioning context; two-way ANOVA $(\mathrm{F} 4,108=5.884, \mathrm{p}=0.0003)$ with Sidak's post hoc tests for conditioning (difference: $0.8941 \pm 4.116,95 \%$ CI: -9.860 to $11.65, \mathrm{p}=$ 0.9928 ), 24 hour (difference: $-0.8004 \pm 4.116,95 \%$ CI: -11.55 to $9.954, p=0.9948$ ), 7 day (difference: $-11.91 \pm 4.116,95 \% \mathrm{CI}:-22.67$ to $-1.160, \mathrm{p}=0.0221$ ), 21 day (difference: $-16.77 \pm 4.116,95 \% \mathrm{CI}:-27.52$ to $-6.013, \mathrm{p}=0.004$ ), and 30 day (difference: $-17.49 \pm$ 4.116, 95\% CI: -16.00 to $-0.3832, \mathrm{p}=0.0002), \mathrm{n}=15$ vehicle and 14 clemastine mice. $\mathbf{c}$, Freezing responses in the similar context; two-way ANOVA $\left(\mathrm{F}_{1,27}=8.277, \mathrm{p}=0.0077\right)$ with Sidak's post hoc tests for 7 day (difference: $0.4158 \pm 3.894,95 \%$ CI: -7.391 to $8.222, p=$ 0.9154 ) and 21 day (difference: $-8.190 \pm 3.894,95 \%$ CI: -28.24 to $-6.733, p=0.401$ ), or across days within vehicle- (difference: $-5.845 \pm 2.078,95 \% \mathrm{CI}:-10.77$ to $-0.9248, \mathrm{p}=$ 0.018 ) and clemastine-treated (difference: $-14.45 \pm 2.151,95 \%$ CI: -19.54 to $-9.357, p<$ 
0.0001) animals, $\mathrm{n}=15$ vehicle and 14 clemastine mice. $\mathbf{d}$, Experiment assessing the effects of continuous vehicle injections/handling, compared against home cage animals; two-way ANOVA $\left(\mathrm{F}_{4,36}=9.926, \mathrm{p}<0.0001\right)$ with Sidak's post hoc tests for conditioning (difference: $-0.6013 \pm 5.615,95 \% \mathrm{CI}:-15.66$ to $14.46, \mathrm{p}>0.9999$ ), 24 hour (difference: $0.2373 \pm$ 5.615, 95\% CI: -14.82 to $15.30, \mathrm{p}>0.9999), 7$ day (difference: $22.20 \pm 5.615,95 \%$ CI: 7.137 to $37.25, \mathrm{p}=0.0014$ ), 21 day (difference: $31.96 \pm 5.615,95 \% \mathrm{CI}: 16.91$ to $47.02, \mathrm{p}<$ 0.0001 ), and 30 day (difference: $26.78 \pm 5.615,95 \% \mathrm{CI}: 11.72$ to $41.83, \mathrm{p}<0.0001$ ), $\mathrm{n}=5$ mice per treatment group. e, Expanded quantification of $\mathrm{Fos}^{+}$cell density following 30-day retrieval sessions; unpaired two-tailed t-tests, PL (difference: $279.0 \pm 43.19,95 \%$ CI: 188.6 to $369.4, \mathrm{t}_{19}=6.459, \mathrm{p}<0.0001$ ), IL (difference: $198.6 \pm 68.81,95 \% \mathrm{CI}: 49.90$ to $347.2, \mathrm{t}_{19}$ $=2.886, \mathrm{p}=0.274$ ), ACC (difference: $127.4 \pm 51.52,95 \% \mathrm{CI}: 18.66$ to $236.1, \mathrm{t}_{19}=2.472, \mathrm{p}$ $=0.0069$ ), BA (difference: $86.21 \pm 19.27,95 \% \mathrm{CI}: 45.87$ to $126.5, \mathrm{t}_{19}=4.473, \mathrm{p}=0.0044$ ), LA (difference: $128.6 \pm 44.43,95 \% \mathrm{CI}: 35.89$ to $221.2, \mathrm{t}_{19}=2.894, \mathrm{p}=0.009$ ), $\mathrm{DG}$ (difference: $268.7 \pm 56.69,95 \%$ CI: 150.0 to $387.3, \mathrm{t}_{19}=4.739,(\mathrm{p}<0.0001), \mathrm{CA} 3$ (difference: $270.5 \pm 77.34,95 \% \mathrm{CI}: 109.2$ to $431.8, \mathrm{t}_{19}=3.497, \mathrm{p}=0.0023$ ), $\mathrm{NR}$ (difference: $110.7 \pm 34.65,95 \%$ CI: 37.59 to $183.8, \mathrm{t}_{19}=3.195, \mathrm{p}=0.0026$ ), $\mathrm{vDG}$ (difference: $153.9 \pm$ 34.77, 95\% CI: 80.51 to $227.2, \mathrm{t}_{19}=4.426, \mathrm{p}=0.0013$ ), vCA3 (difference: $139.2 \pm 36.88$, $95 \%$ CI: 61.42 to $217.0, \mathrm{t}_{19}=3.775, \mathrm{p}=0.0019$ ), vPAG (difference: $193.0 \pm 48.67,95 \% \mathrm{CI}$ : 90.28 to $295.6, \mathrm{t}_{19}=3.965, \mathrm{p}=0.0017$ ), $\mathrm{SSC}$ (difference: $79.25 \pm 55.73,95 \% \mathrm{CI}:-38.33$ to $\left.196.8, \mathrm{t}_{19}=1.422, \mathrm{p}=0.1731\right), \mathrm{n}=13$ vehicle and 8 clemastine mice. $\mathbf{f}$, Within-treatment group comparisons of $\mathrm{Fos}^{+}$cell density between the PL and IL; unpaired two-tailed t-tests, vehicle (difference: $80.43 \pm 48.18,95 \% \mathrm{CI}:-20.78$ to $181.6, \mathrm{t}_{18}=1.67, \mathrm{p}=0.3657$ ), $\mathrm{n}=13$ PL- and 7 IL-sampled mice, clemastine (difference: $-24.05 \pm 65.62,95 \% \mathrm{CI}:-162.5$ to $\left.114.4, \mathrm{t}_{17}=0.3666, \mathrm{p}=0.7185\right), \mathrm{n}=8 \mathrm{PL}$ - and $11 \mathrm{IL}$-sampled mice. Freezing responses for experimental timeline described in (a) for home cage $(\mathbf{g})$, no shock $(\mathbf{h})$, and immediate shock (i) animals; (g) two-way ANOVA $\left(\mathrm{F}_{3,24}=2.217, \mathrm{p}=0.1122\right)(\mathbf{h})$ two-way ANOVA $\left(\mathrm{F}_{4,32}=\right.$ $2.674, \mathrm{p}=0.0496)$ (i) two-way ANOVA $\left(\mathrm{F}_{4,32}=0.7811, \mathrm{p}=0.5496\right)$; for $\mathrm{g}-\mathrm{I}, \mathrm{n}=5$ animals per condition. $\mathbf{j}$, Extended experimental timeline for clemastine injections in Myrf icKO animals. k, Individual fear expression for vehicle- and clemastine-treated Myrf icKO animals represented in $(\mathbf{j})$; two-way ANOVA $\left(\mathrm{F}_{4,48}=1.357, \mathrm{p}=0.2629\right), \mathrm{n}=6$ vehicle and 8 clemastine mice. I, Quantification of the $\mathrm{z}$-scored mean $\Delta \mathrm{F} / \mathrm{F}$ during pre- and post-bout transitions at 30 days for vehicle- (left) and clemastine-treated (right) animals; paired twotailed t-tests comparing pre- and post-transition for vehicle (difference: $1.075 \pm 0.1560,95 \%$ CI: 0.7685 to $1.382, \mathrm{t}_{78}=6.894, \mathrm{p}<0.0001$ ) and clemastine (difference: $0.8792 \pm 0.1548$, $95 \%$ CI: 0.5745 to $\left.1.184, \mathrm{t}_{78}=5.678, \mathrm{p}<0.0001\right)$. $\mathrm{m}$, Quantification of the $\mathrm{z}$-scored mean $\Delta \mathrm{F} / \mathrm{F}$ of the pre- and post-bout transition for vehicle- and clemastine-treated animals; unpaired two-tailed t-test (difference: $-0.1963 \pm 0.2099,95 \% \mathrm{CI}:-0.6094$ to $0.2168, \mathrm{t}_{78}=$ $0.9352, \mathrm{p}=0.3504)$. For $1-\mathrm{m}, \mathrm{n}=10$ bouts per animal, 4 animals per treatment group. For box-and-whisker plots, the center, boxes, and whiskers represent the median, interquartile range, and the $10^{\text {th }}$ and $90^{\text {th }}$ percentiles. For dot plots, data are presented as mean \pm SEM, with asterisks indicating the following p-value ranges: $* \leq 0.05, * * \leq 0.01$, $* * * \leq 0.001$, $* * * * \leq 0.0001$. 


\section{Supplementary Material}

Refer to Web version on PubMed Central for supplementary material.

\section{Acknowledgements}

We are grateful to K. Hoi for providing reagents, insightful discussion, technical assistance and comments on the manuscript. We also thank members of the Chan and Kheirbek laboratories for technical assistance and scientific discussion, and V. Sohal, S. Pleasure, K. Poskanzer and A. Molofsky for insightful discussions. This work was supported by the National Institutes of Health/National Institute of Neurological Disorders and Stroke (R01NS062796, R01NS097428 and R01NS095889), the Adelson Medical Research Foundation (ANDP grant A130141) and the Rachleff Family Endowment to J.R.C., and the NIMH (R01 MH108623, R01 MH111754 and R01 MH117961), a Weill Scholar Award, the Pew Charitable Trusts, the Esther A. and Joseph Klingenstein Fund, and an IMHRO/One Mind Rising Star Award to M.A.K.

\section{References}

1. Hill R, Patel K, Goncalves C, Grutzendler J \& Nishiyama A Modulation of oligodendrocyte generation during a critical temporal window after NG2 cell division. Nat. Neurosci 17, 1518-1527 (2014). [PubMed: 25262495]

2. Hill R, Li AM \& Grutzendler J Lifelong cortical myelin plasticity and age-related degeneration in the live mammalian brain. Nat. Neurosci 21, 683-695 (2018). [PubMed: 29556031]

3. Hughes E, Kang S, Fukaya M \& Bergles D Oligodendrocyte progenitors balance growth with selfrepulsion to achieve homeostasis in the adult brain. Nat. Neurosci 16, 668-676 (2013). [PubMed: 23624515]

4. Hughes E, Orthmann-Murphy J, Langseth A \& Bergles D Myelin remodeling through experiencedependent oligodendrogenesis in the adult somatosensory cortex. Nat. Neurosci 21, 696-706 (2018). [PubMed: 29556025]

5. Kang SH, Fukaya M, Yang JK, Rothstein JD \& Bergles DE NG2+ CNS glial progenitors remain committed to the oligodendrocyte lineage in postnatal life and following neurodegeneration. Neuron 68, 668-681 (2010). [PubMed: 21092857]

6. Young $\mathrm{K}$ et al. Oligodendrocyte dynamics in the healthy adult CNS: evidence for myelin remodeling. Neuron 77, 873-885 (2013). [PubMed: 23473318]

7. Bergles DE, Roberts JD, Somogyi P \& Jahr CE Glutamatergic synapses on oligodendrocyte precursor cells in the hippocampus. Nature 405, 187-191 (2000). [PubMed: 10821275]

8. Lin S \& Bergles D Synaptic signaling between GABAergic interneurons and oligodendrocyte precursor cells in the hippocampus. Nat. Neurosci 7, 24-32 (2004). [PubMed: 14661022]

9. Gibson E et al. Neuronal activity promotes oligodendrogenesis and adaptive myelination in the mammalian brain. Science 344, 1252304 (2014). [PubMed: 24727982]

10. Mitew $\mathrm{S}$ et al. Pharmacogenetic stimulation of neuronal activity increases myelination in an axonspecific manner. Nat. Commun 9, 306 (2018). [PubMed: 29358753]

11. Geraghty A et al. Loss of adaptive myelination contributes to methotrexate chemotherapy-related cognitive impairment. Neuron 103, 250-265 (2019). [PubMed: 31122677]

12. Etxeberria A et al. Dynamic modulation of myelination in response to visual stimuli alters optic nerve conduction velocity. J. Neurosci 36, 6937-6948 (2016). [PubMed: 27358452]

13. Makinodan M, Rosen KM, Ito S \& Corfas G A critical period for social experience-dependent oligodendrocyte maturation and myelination. Science 337, 1357-1360 (2012). [PubMed: 22984073]

14. Mangin JM, Li P, Scafidi J \& Gallo V Experience-dependent regulation of NG2 progenitors in the developing barrel cortex. Nat. Neurosci 15, 1192-1194 (2012). [PubMed: 22885848]

15. McKenzie IA et al. Motor skill learning requires active central myelination. Science 346, 318-322 (2014). [PubMed: 25324381]

16. Liu $\mathrm{J}$ et al. Impaired adult myelination in the prefrontal cortex of socially isolated mice. Nat. Neurosci 36, 957-962 (2012). 
17. Xiao L et al. Rapid production of new oligodendrocytes is required in the earliest stages of motorskill learning. Nat. Neurosci 19, 1210-1217 (2016). [PubMed: 27455109]

18. Tripathi RB et al. Remarkable stability of myelinating oligodendrocytes in mice. Cell Rep 21, 316323 (2017). [PubMed: 29020619]

19. Pan S \& Chan JR Regulation and dysregulation of axon infrastructure by myelinating glia. J. Cell Biol 216, 3903-3916 (2017). [PubMed: 29114067]

20. de Hoz L \& Simons M The emerging functions of oligodendrocytes in regulating neuronal network behaviour. BioEssays 37, 60-69 (2015). [PubMed: 25363888]

21. Shalev A, Liberzon I \& Marmar C Post-traumatic stress disorder. N. Engl. J. Med 376, 2459-2469 (2017). [PubMed: 28636846]

22. Tovote P, Fadok JP \& Lüthi A Neuronal circuits for fear and anxiety. Nat. Rev. Neurosci 16, $317-$ 331 (2015). [PubMed: 25991441]

23. Cowansage KK et al. Direct reactivation of a coherent neocortical memory of context. Neuron 84 , 432-441 (2014). [PubMed: 25308330]

24. DeNardo LA et al. Temporal evolution of cortical ensembles promoting remote memory retrieval. Nat. Neurosci 22, 460-469 (2019). [PubMed: 30692687]

25. Kitamura $\mathrm{T}$ et al. Engrams and circuits crucial for systems consolidation of a memory. Science 356, 73-78 (2017). [PubMed: 28386011]

26. Tanaka KZ Cortical representations are reinstated by the hippocampus during memory retrieval. Neuron 84, 347-354 (2014). [PubMed: 25308331]

27. Vetere $\mathrm{G}$ et al. Chemogenetic interrogation of a brain-wide fear memory network in mice. Neuron 94, 363-374 (2017). [PubMed: 28426969]

28. Frankland PW \& Bontempi B The organization of recent and remote memories. Nat. Rev. Neurosci 6, 119-130 (2005). [PubMed: 15685217]

29. Tonegawa S, Morrissey M \& Kitamura $T$ The role of engram cells in the systems consolidation of memory. Nat. Rev. Neurosci 19, 485-498 (2018). [PubMed: 29970909]

30. Emery B et al. Myelin gene regulatory factor is a critical transcriptional regulator required for CNS myelination. Cell 138, 172-185 (2009). [PubMed: 19596243]

31. Giustino TF, Fitzgerald PJ \& Maren S Fear expression suppresses medial prefrontal cortical firing in rats. PLoS One 11, e0165256 (2016). [PubMed: 27776157]

32. Halladay LR \& Blair HT Distinct ensembles of medial prefrontal cortex neurons are activated by threatening stimuli that elicit excitation vs. inhibition of movement. J. Neurophysiol 114, 793-807 (2015). [PubMed: 25972588]

33. Mei $\mathrm{F}$ et al. Micropillar arrays as a high-throughput screening platform for therapeutics in multiple sclerosis. Nat. Med 20, 954-960 (2014). [PubMed: 24997607]

34. Mei $\mathrm{F}$ et al. Accelerated remyelination during inflammatory demyelination prevents axonal loss and improves functional recovery. eLife 5, e18246 (2016). [PubMed: 27671734]

35. Green AJ, Gelfand JM, Cree BA \& Lancet B-C Clemastine fumarate as a remyelinating therapy for multiple sclerosis (ReBUILD): a randomised, controlled, double-blind, crossover trial. Lancet 390, 2481-2489 (2017). [PubMed: 29029896]

36. Liu J et al. Clemastine enhances myelination in the prefrontal cortex and rescues behavioral changes in socially isolated mice. J. Neurosci 36, 957-962 (2016). [PubMed: 26791223]

37. Wang $\mathrm{F}$ et al. Enhancing oligodendrocyte myelination rescues synaptic loss and improves functional recovery after chronic hypoxia. Neuron 99, 689-701 (2018). [PubMed: 30078577]

38. Guo $\mathrm{N}$ et al. Dentate granule cell recruitment of feedforward inhibition governs engram maintenance and remote memory generalization. Nat. Med 24, 438-449 (2018). [PubMed: 29529016]

39. Freeman SA et al. Acceleration of conduction velocity linked to clustering of nodal components precedes myelination. Proc. Natl Acad. Sci. USA 112, 321-328 (2015).

40. Fünfschilling U et al. Glycolytic oligodendrocytes maintain myelin and long-term axonal integrity. Nature 485, 517-522 (2012). [PubMed: 22622581]

41. Lee $Y$ et al. Oligodendroglia metabolically support axons and contribute to neurodegeneration. Nature 487, 443-448 (2012). [PubMed: 22801498] 
42. Xin W et al. Oligodendrocytes support neuronal glutamatergic transmission via expression of glutamine synthetase. Cell Rep 27, 2262-2271 (2019). [PubMed: 31116973]

43. Frankland PW, Bontempi B, Talton LE, Kaczmarek L \& Silva AJ The involvement of the anterior cingulate cortex in remote contextual fear memory. Science 304, 881-883 (2004). [PubMed: 15131309]

44. Maviel T, Durkin T, Menzaghi F \& Bontempi B Sites of neocortical reorganization critical for remote spatial memory. Science 305, 96-99 (2004). [PubMed: 15232109]

45. Benchenane $\mathrm{K}$ et al. Coherent theta oscillations and reorganization of spike timing in the hippocampal-prefrontal network upon learning. Neuron 66, 921-936 (2010). [PubMed: 20620877]

46. Karalis $\mathrm{N}$ et al. 4-Hz oscillations synchronize prefrontal-amygdala circuits during fear behavior. Nat. Neurosci 19, 605-612 (2016). [PubMed: 26878674]

47. Xia F et al. Parvalbumin-positive interneurons mediate neocortical-hippocampal interactions that are necessary for memory consolidation. eLife 6, e27868 (2017). [PubMed: 28960176]

48. Pajevic S, Basser PJ \& Fields RD Role of myelin plasticity in oscillations and synchrony of neuronal activity. Neuroscience 276, 135-147 (2014). [PubMed: 24291730]

49. Steadman PE et al. Disruption of oligodendrogenesis impairs memory consolidation in adult mice. Neuron 10.1016/j.neuron.2019.10.013 (2019).

50. Chao L, Tosun D, Woodward S, Kaufer D \& Neylan T Preliminary evidence of increased hippocampal myelin content in veterans with posttraumatic stress disorder. Front. Behav. Neurosci 9, 333 (2015). [PubMed: 26696852]

51. Chen TW et al. Ultrasensitive fluorescent proteins for imaging neuronal activity. Nature 499, $295-$ 300 (2013). [PubMed: 23868258]

52. Schindelin J et al. Fiji: an open-source platform for biological-image analysis. Nat. Methods 9 , 676-682 (2012). [PubMed: 22743772] 


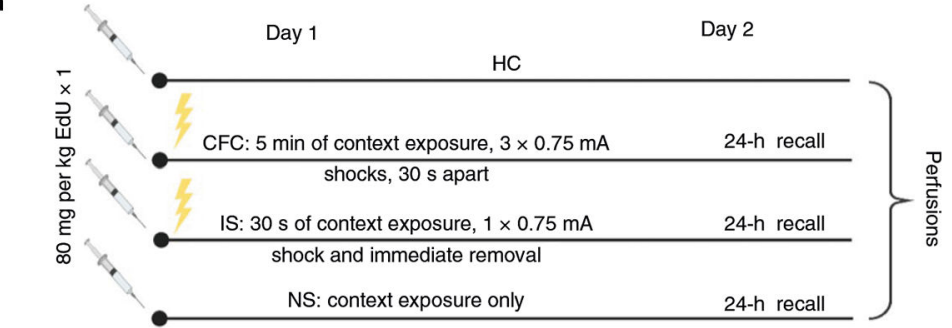

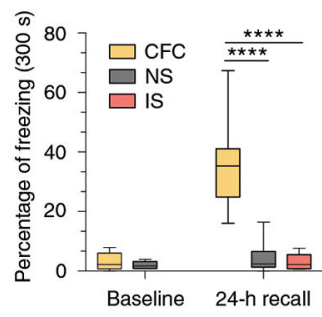

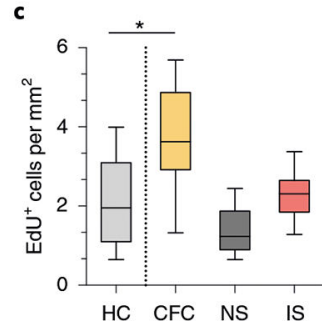

d

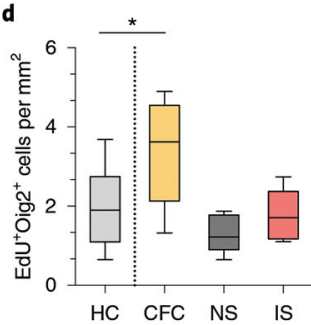

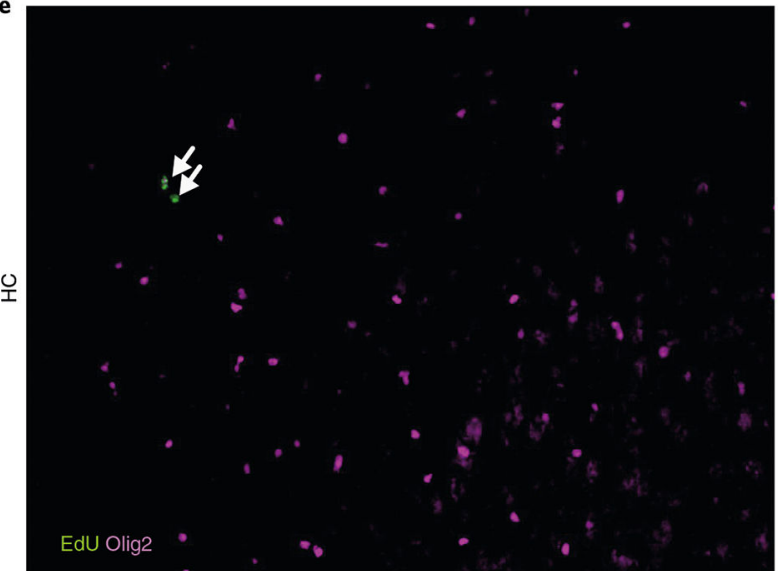

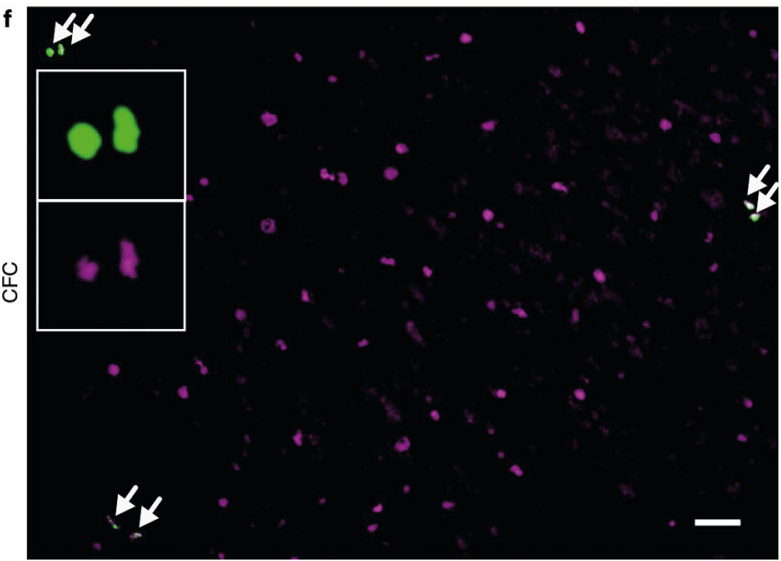

Fig. 1 . Fear learning induces oPC proliferation in the mPFC.

a, Experimental paradigm for EdU injections and contextual fear conditioning. b, Freezing responses pre-shock and during 24-h recall for home cage (HC), contextual fear conditioned (CFC), no shock (NS) and immediate shock (IS) groups. One-way ANOVA $\left(F_{2,20}=28.35, P\right.$ $<0.0001$ ) with Sidak's post hoc tests comparing CFC versus NS (difference: $-31.19 \pm$ 6.179; 95\% CI: -44.54 to $-17.84 ; P<0.0001$ ) and CFC versus IS (difference: $-32.94 \pm$ 5.536; $95 \%$ CI: -44.81 to $-21.07 ; P<0.0001)$. c, Total EdU ${ }^{+}$cell density in the mPFC of HC, CFC, NS and IS animals. One-way ANOVA $\left(F_{3,26}=7.238, P=0.0012\right)$ with Sidak's post hoc tests comparing $\mathrm{HC}$ versus CFC (difference: $-1.616 \pm 0.5212 ; 95 \% \mathrm{CI}:-2.946$ to $-0.2868 ; P=0.0137$ ), $\mathrm{HC}$ versus NS (difference: $0.7080 \pm 0.5382 ; 95 \% \mathrm{CI}:-0.6654$ to $2.081 ; P=0.4878$ ) and $\mathrm{HC}$ versus IS (difference: $-0.1819 \pm 0.5212 ; 95 \% \mathrm{CI}:-1.512$ to 1.148; $P=0.9803)$. d, EdU ${ }^{+}$Olig2 ${ }^{+}$cell density in the mPFC of HC, CFC, NS and IS animals. One-way ANOVA $\left(F_{3,26}=7.672, P=0.0008\right)$ with Sidak's post hoc tests 
comparing HC versus CFC (difference: $-1.393 \pm 0.47 ; 95 \%$ CI: -2.592 to $-0.1933 ; P=$ 0.0192 ), HC versus NS (difference: $0.7474 \pm 0.4854,95 \%$ CI: -0.4912 to $1.986 ; P=0.3544$ ) and HC versus IS (difference: $0.1734 \pm 0.47 ; 95 \% \mathrm{CI}:-1.026$ to $1.373 ; P=0.9769$ ) at $24 \mathrm{~h}$ post-conditioning. e,f, Representative images of EdU (green) and Olig2 (magenta) staining in the $\mathrm{mPFC}$ of $\mathrm{HC}(\mathbf{e})$ and $\mathrm{CFC}(\mathbf{f})$ animals, with arrows indicating colocalized EdU ${ }^{+} \mathrm{Olig} 2^{+}$ cells; inset depicts separate color channels for representative $\mathrm{EdU}^{+}$and Olig2 ${ }^{+}$cells. For all panels, $n=7$ mice (HC), 8 mice (CFC), 7 mice (NS) or 8 mice (IS). Scale bar, $50 \mu \mathrm{m}$. For box-and-whisker plots, the center, boxes and whiskers represent the median, interquartile range, and the 10th and 90th percentiles, respectively. ${ }^{*} P \leq 0.05$, **** $P \leq 0.0001$. 
a

n.m.

(1) -

EdU injections $\times 5,80 \mathrm{mg}$ per $\mathrm{kg}$

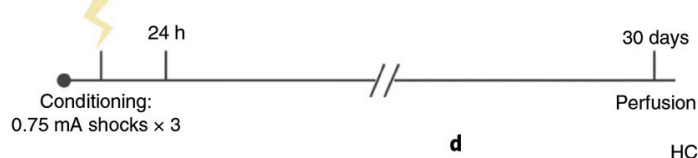

HC
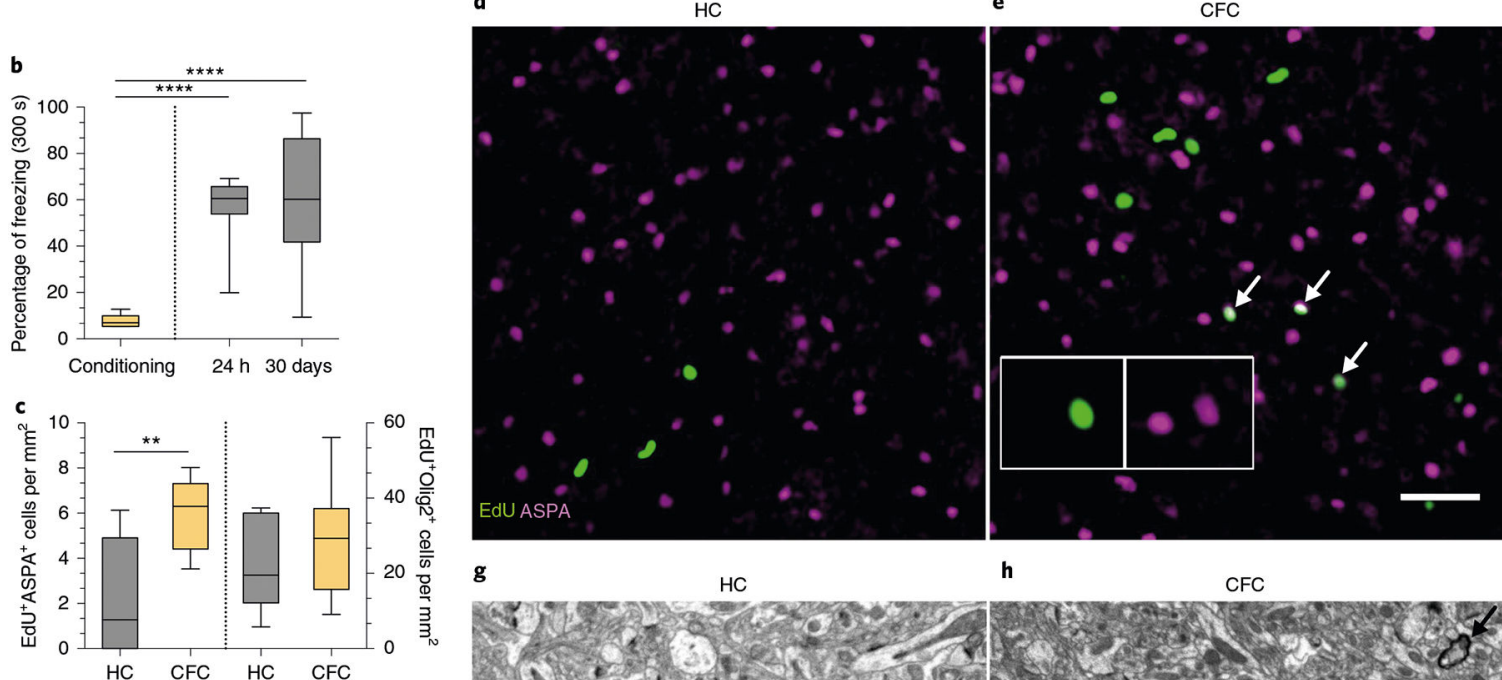

g

$\mathrm{HC}$

h

CFC

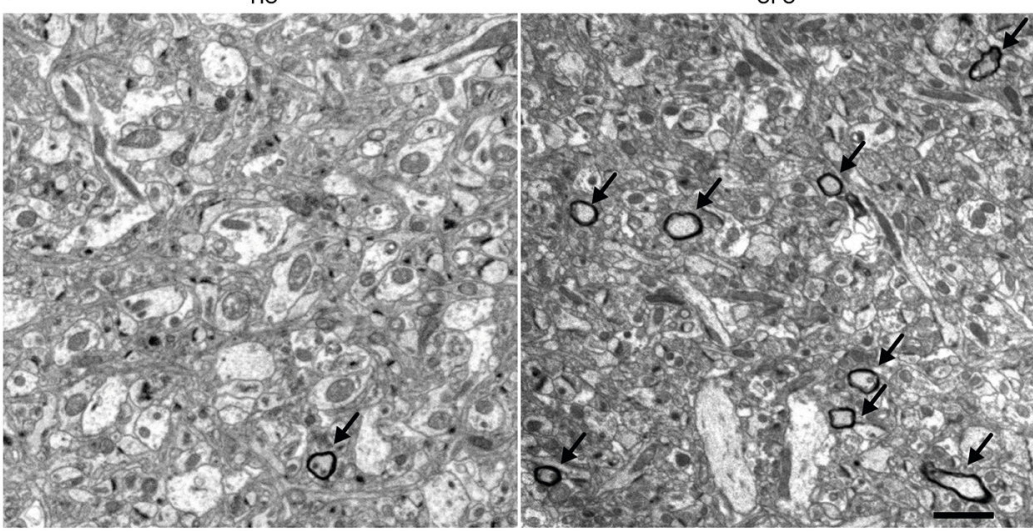

Fig. 2 |. Fear learning experience induces oPC maturation into myelinating oLs in the mPFC. a, Experimental paradigm for EdU injections and contextual fear conditioning. b, Freezing responses during pre-conditioning and 24-h and 30-day retrieval sessions. One-way ANOVA $\left(F_{2,21}=19.42, P<0.0001\right)$ with Sidak's post hoc tests comparing conditioning versus $24 \mathrm{~h}$ (difference: $-48.53 \pm 9.402 ; 95 \% \mathrm{CI}:-71.17$ to $-25.89 ; P<0.0001$ ) and conditioning versus 30 days (difference: $-52.71 \pm 9.402 ; 95 \%$ CI: -75.34 to $-30.07 ; P<0.0001$ ). c, EdU ${ }^{+}$ASPA ${ }^{+}$(left bars) and $\mathrm{EdU}^{+} \mathrm{Olig} 2^{+}$(right bars) density in the $\mathrm{mPFC}$ of $\mathrm{HC}$ and $\mathrm{CFC}$ animals at 30 days post-conditioning. Unpaired two-tailed $t$-tests for $\mathrm{HC}$ versus $\mathrm{CFC}$ for $\mathrm{EdU}^{+} \mathrm{ASPA}^{+}$ (difference: $3.629 \pm 1.125 ; 95 \%$ CI: 1.199 to $6.060 ; t_{13}=3.226, P=0.0066$ ) and for EdU ${ }^{+}$Olig2 ${ }^{+}$(difference: $7.555 \pm 6.944 ;$; 95 CI: -7.446 to $22.56 ; t_{13}=1.088, P=0.2963$ ) cell density. d,e, Representative images of EdU (green) and ASPA (magenta) in the mPFC of HC (d) and CFC (e) animals, with arrows indicating colocalized $\mathrm{EdU}^{+} \mathrm{ASPA}^{+}$cells; inset depicts separate color channels for representative $\mathrm{EdU}^{+} \mathrm{ASPA}^{+}$cells. f, Quantification of myelinated axon density per field of view in the mPFC. Unpaired two-tailed $t$-tests comparing HC versus CFC (difference: $23.07 \pm 5.882 ; 95 \%$ CI: 10.95 to $35.18 ; t_{25}=3.922, P=0.0006$ ). $\mathbf{g , h}, \mathbf{h}$, Representative electron micrographs of myelinated axons (arrows) in mPFC gray matter 
for HC (g) and CFC (h) animals. For b-c, $n=7$ mice (HC) or 8 mice (CFC); for $\mathbf{f}, n=5$ mice (HC) or 4 mice (CFC), 3 fields of view per mouse. Scale bars, $50 \mu \mathrm{m}(\mathbf{d}-\mathbf{e})$ or $2 \mu \mathrm{m}(\mathbf{g}-$ h). For box-and-whisker plots, the center, boxes and whiskers represent the median, interquartile range, and the 10th and 90th percentiles, respectively. $* * P \leq 0.01$, *** $P \leq$ $0.001, * * * * P \leq 0.0001$. 

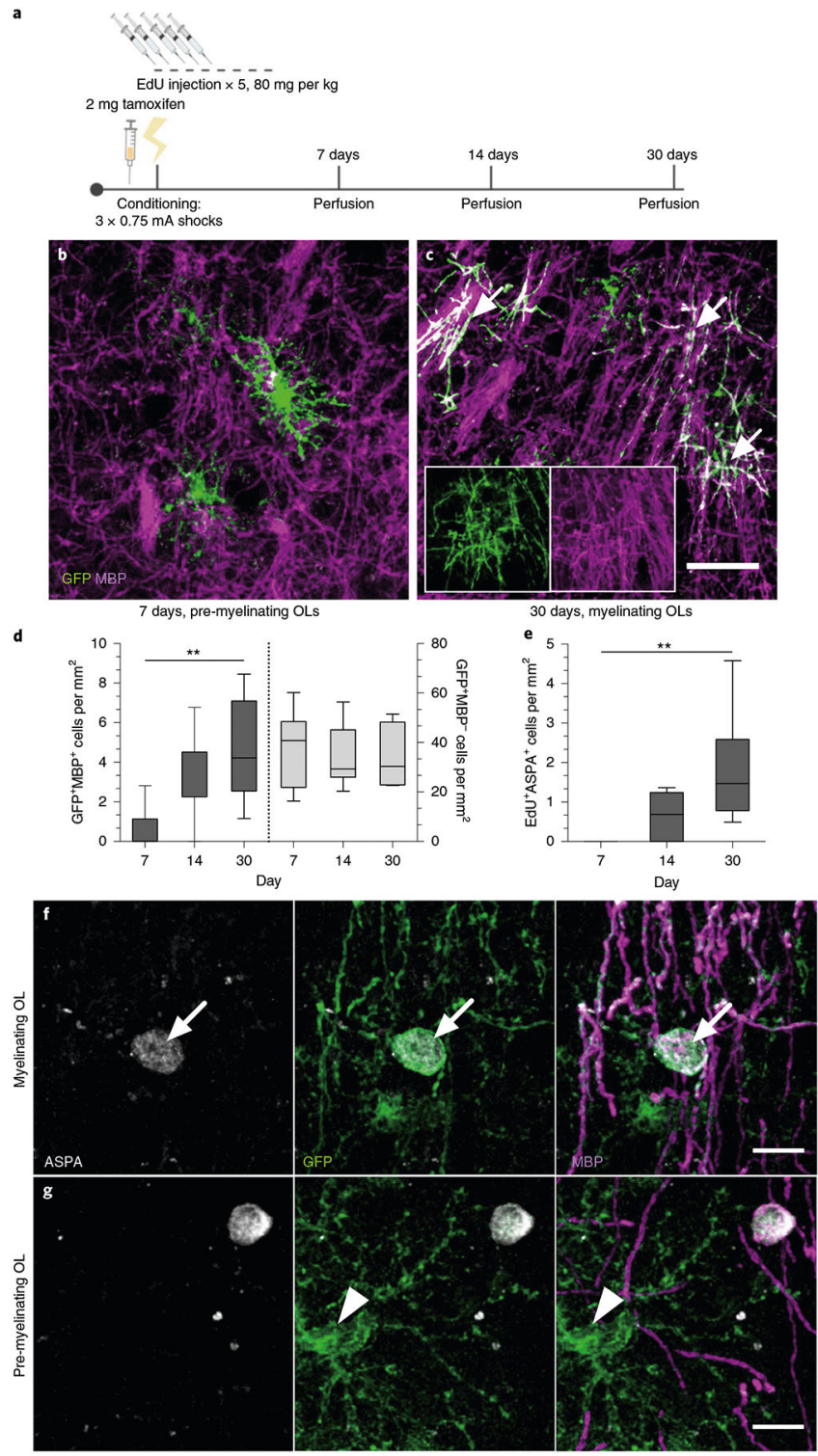

Fig. 3 | oPC maturation into myelinating oLs occurs over several weeks in adult gray matter. a, Experimental paradigm for tamoxifen injections, EdU injections, contextual fear conditioning and perfusions. b,c, Representative images of $\mathrm{GFP}^{+}$(green) and $\mathrm{MBP}^{-}$ (magenta) pre-myelinating OLs at 7 days post-tamoxifen (b) and $\mathrm{GFP}^{+} \mathrm{MBP}^{+}$myelinating OLs (arrows) at 30 days post-tamoxifen (c) in the $\mathrm{mPFC}$ of CFC mice; inset depicts separate color channels for representative $\mathrm{GFP}^{+} \mathrm{MBP}^{+}$cells. d, Quantification of $\mathrm{GFP}^{+} \mathrm{MBP}^{+}$(left bars) and $\mathrm{GFP}^{+} \mathrm{MBP}^{-}$(right bars) cell density in the $\mathrm{mPFC}$ over time. For $\mathrm{GFP}^{+} \mathrm{MBP}^{+}$ density, one-way ANOVA $\left(F_{2,19}=6.586, P=0.0067\right)$ with Sidak's post hoc tests comparing 7 versus 14 days (difference: $-2.695 \pm 1.070 ; 95 \%$ CI: -5.292 to $-0.09785 ; P=0.0614$ ) and 7 versus 30 days (difference: $-4.045 \pm 1.129 ; 95 \% \mathrm{CI}:-6.787$ to $-1.304 ; P=0.004)$ ). For $\mathrm{GFP}^{+} \mathrm{MBP}^{-}$density, one-way ANOVA $\left(F_{2,21}=0.07572, P=0.9274\right)$ with Sidak's post hoc tests comparing 7 versus 14 days (difference: $2.354 \pm 6.908$; 95\% CI: -14.41 to $19.12 ; P=$ 0.9308 ) and 7 versus 30 days (difference: $2.540 \pm 7.292$; $95 \%$ CI: -15.16 to $20.24 ; P=$ 
0.9279). e, $\mathrm{EdU}^{+} \mathrm{ASPA}^{+}$density in the mPFC over time. One-way ANOVA $\left(F_{2,18}=7.209, P\right.$ $=0.005$ ) with Sidak's post hoc tests comparing 7 versus 14 days (difference: $-0.5957 \pm$ $0.5125 ; 95 \%$ CI: -1.846 to $0.6543 ; P=0.4529$ ) and 7 versus 30 days (difference: $-1.817 \pm$ 0.4975 ; 95\% CI: -3.031 to $-0.6038 ; P=0.0036)$. $\mathbf{f}, \mathbf{g}$, Representative images of $\mathrm{GFP}^{+} \mathrm{MBP}$ ${ }^{+} \mathrm{ASPA}^{+}$myelinating $\mathrm{OLs}$ (f, arrows) and $\mathrm{GFP}^{+} \mathrm{MBP}^{-} \mathrm{ASPA}^{-}$pre-myelinating OLs (g, arrowheads) in the mPFC at 30 days post-conditioning. For all panels, $n=6$ mice ( 7 days), 9 mice (14 days) or 7 mice (30 days). Scale bars, $50 \mu \mathrm{m}$ (b,c) or $10 \mu \mathrm{m}(\mathbf{f}, \mathbf{g})$. For box-andwhisker plots, the center, boxes and whiskers represent the median, interquartile range, and the 10th and 90th percentiles, respectively. $* * P \leq 0.01$. 

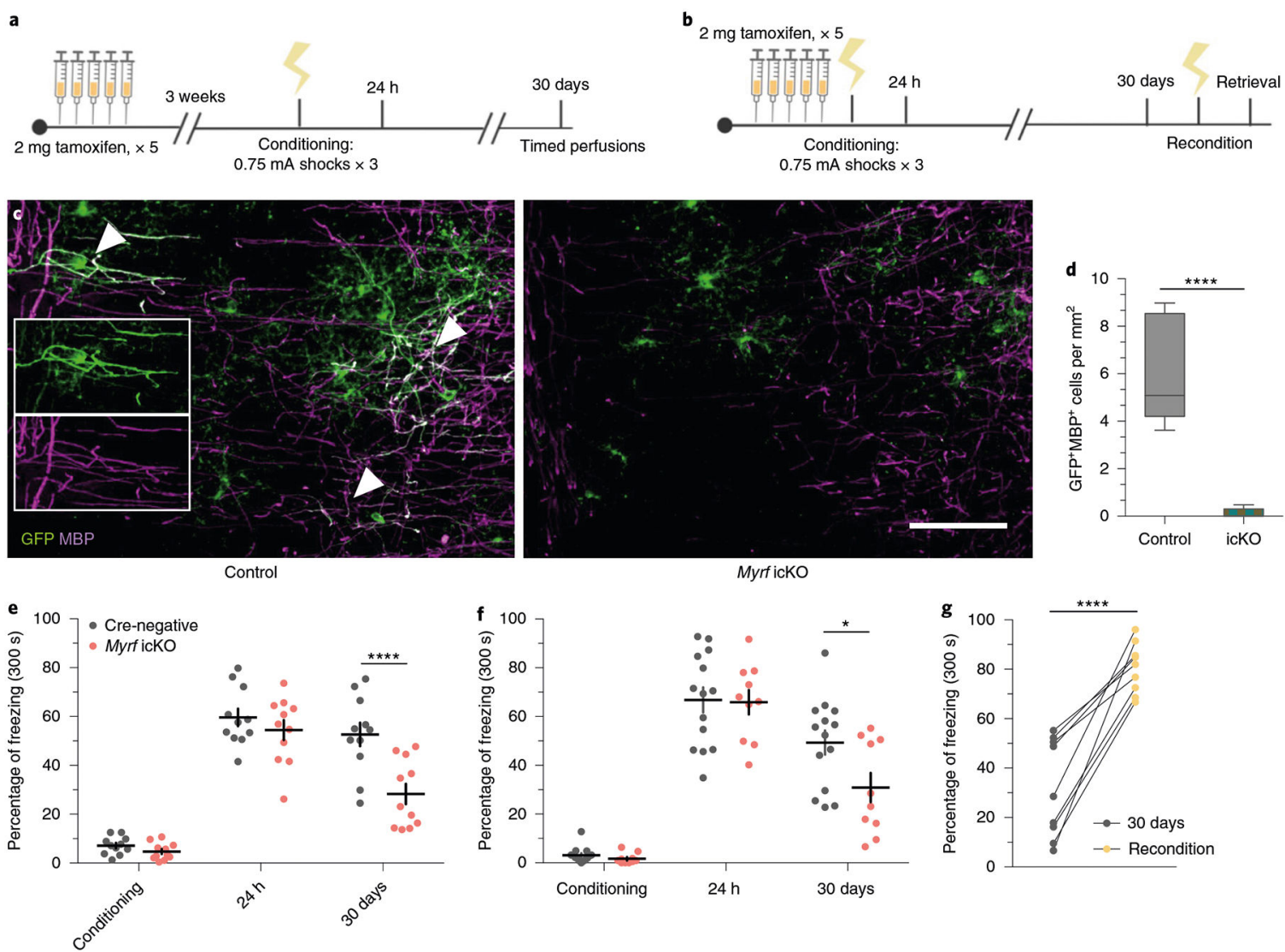

Fig. 4 |. inhibition of new myelin formation impairs remote fear memory recall.

a, Experimental paradigm for contextual fear conditioning with tamoxifen administered 3 weeks before conditioning. b, Experimental paradigm for contextual fear conditioning with tamoxifen administered 5 days before conditioning. c, Representative images of GFP (green) and MBP (magenta) staining in the mPFC of $M y r f^{\text {loxP/+ }}$;tau-mGFPloxP/loxP ${ }^{\text {;NG }} 2 \mathrm{CreERT}^{+}$ (left, control) animals and in $M y r f^{\text {doxP/loxP }}$;tau-MGFP ${ }^{\text {loxP/loxP }} ; \mathrm{NG}^{2} \mathrm{CreERT}^{+}$(right, $\mathrm{Myrf}$ icKO) animals 30 days post-conditioning; inset depicts separate color channels for representative $\mathrm{GFP}^{+} \mathrm{MBP}^{+}$cells. d, Quantification of $\mathrm{GFP}^{+} \mathrm{MBP}^{+}$cell density in the mPFC of control and Myrf icKO animals 30 days post-conditioning; $n=6$ mice per condition (control, MyrficKO), unpaired two-tailed $t$-test (difference: $-5.806 \pm 0.9062,95 \% \mathrm{CI}$ : -7.825 to $\left.-3.786, t_{10}=6.406, P<0.0001\right)$. e,f, Individual fear expression over time for $M y r f$ icKO and control animals administered tamoxifen 3 weeks (e) and 5 days (f) before conditioning. For e, two-way ANOVA $\left(F_{4,80}=3.437, P=0.0121\right)$ with Sidak's post hoc tests comparing Cre-negative versus $M y r f$ icKO during conditioning (difference: $-2.416 \pm$ 5.790; $95 \%$ CI: -17.58 to $12.74 ; P=0.9965$ ), 24 h (difference: $-5.169 \pm 5.790 ; 95 \%$ CI: -20.33 to $9.991 ; P=0.9040$ ) and 30 days (difference: $-24.37 \pm 5.790 ; 95 \% \mathrm{CI}:-39.53$ to -9.209; $P=0.0003), n=11$ mice per genotype. For $\mathbf{f}$, two-way ANOVA $\left(F_{4,80}=1.435, P=\right.$ 0.2291) with Sidak's post hoc tests comparing Cre-negative versus MyrficKO during conditioning (difference $1.467 \pm 7.398 ; 95 \% \mathrm{CI}:-13.19$ to $16.13 ; P=0.8431$ ), $24 \mathrm{~h}$ (difference: $0.9199 \pm 7.398 ; 95 \% \mathrm{CI}:-13.74$ to $15.58 ; P=0.9013$ ) and 30 days (difference: $18.43 \pm 7.398 ; 95 \% \mathrm{CI}: 3.767$ to $33.09 ; P=0.0142), n=14$ Cre-negative and $10 \mathrm{Myrf}$ icKO 
mice. g, Freezing responses for Myrf icKO animals from the cohort in $\mathbf{f} 24 \mathrm{~h}$ after being reconditioned 31 days post-conditioning. Paired two-tailed $t$-test (difference: $48.76 \pm 6.624$; 95\% CI: 33.48 to $\left.64.04 ; t_{8}=7.361, P<0.0001\right)$. Scale bar, $50 \mu \mathrm{m}$. For box-and-whisker plots, the center, boxes and whiskers represent the median, interquartile range, and the 10th and 90 th percentiles, respectively. For dot plots, data are presented as the mean \pm s.e.m. ${ }^{*} P \leq$ $0.05, * * * * P \leq 0.0001$. 

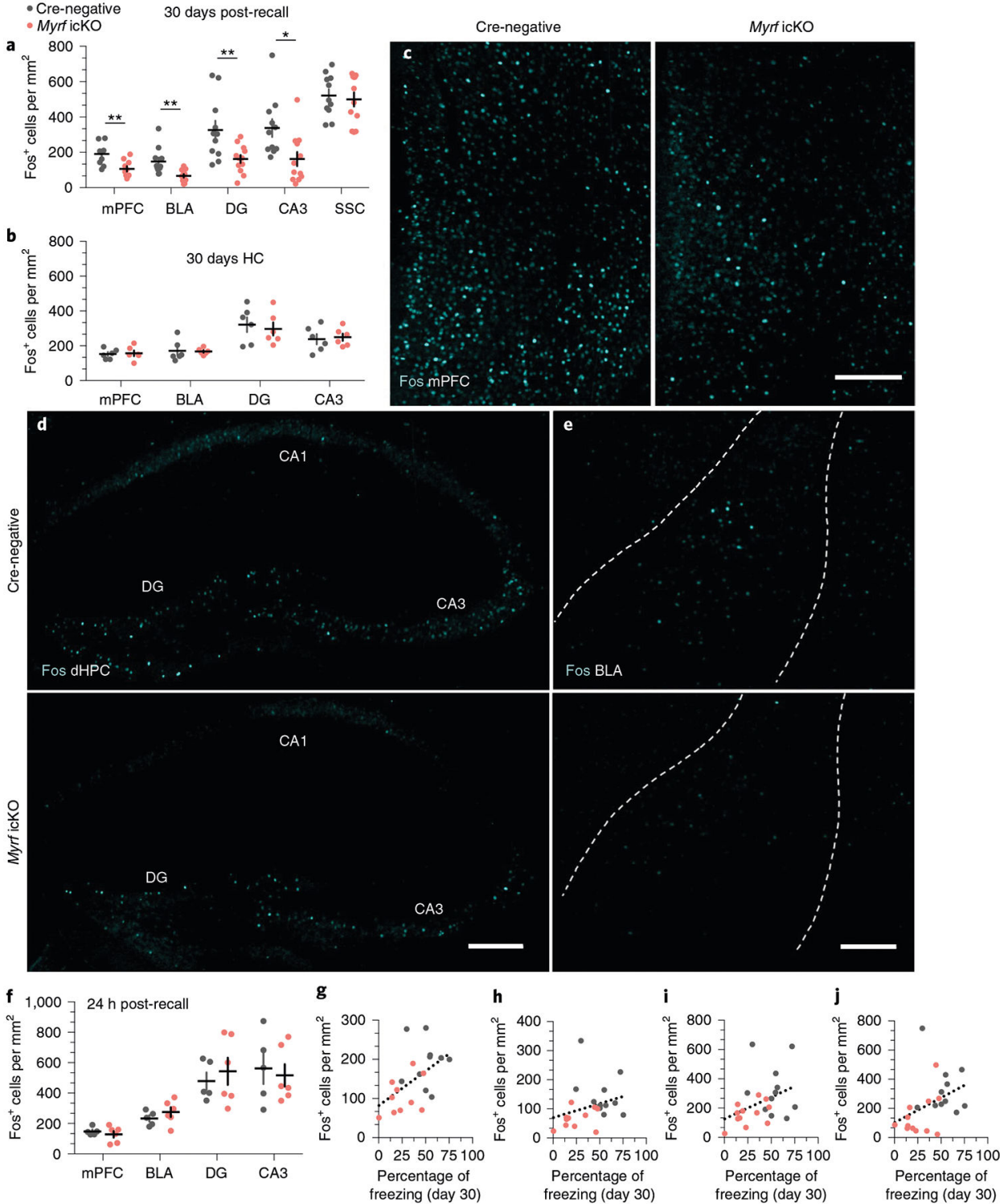

Fig. 5 |. immediate early gene expression following remote recall is impaired in the absence of new myelin formation.

a, Quantification of Fos ${ }^{+}$cell density across brain regions following 30-day retrieval sessions in Myrf icKO versus Cre-negative controls (the key to the chart also applies to b,fj). Unpaired two-tailed $t$-tests, mPFC (difference: $84.27 \pm 23.89$; 95\% CI: -34.09 to 134.5 ; $t_{21}=3.528, P=0.0024$ ), BLA (difference: $80.55 \pm 24.87$; $95 \%$ CI: 28.66 to $132.4 ; t_{21}=$ 3.238, $P=0.0041$ ), DG (difference: $163.5 \pm 55.29,95 \% \mathrm{CI}: 48.50$ to $278.5, t_{21}=2.957, P=$ 0.0075 ), CA3 (difference: $175.4 \pm 63.83$; $95 \%$ CI: 42.70 to $308.2 ; t_{21}=2.749, P=0.012$ ), SSC (difference: $21.86 \pm 53.48$; 95\% CI: -89.35 to $133.1 ; t_{21}=0.4088, P=0.6868$ ), $n=12$ Cre-negative and 11 Myrf icKO mice. b, Quantification of baseline $\mathrm{HC} \mathrm{Fos}^{+}$cell density across brain regions in Myrf icKO versus Cre-negative controls. Unpaired two-tailed $t$-tests, mPFC (difference: $4.591 \pm 19.85$; $95 \%$ CI: -39.64 to $48.82 ; t_{10}=0.2313, P=0.8218$ ), BLA (difference: $-3.475 \pm 25.49$; $95 \%$ CI: -60.28 to $53.33 ; t_{10}=0.1363, P=0.8943$ ), DG (difference: $-24.73 \pm 56.36$; $95 \% \mathrm{CI}:-150.3$ to $100.8 ; t_{10}=0.4387, P=0.6702$ ), CA3 
(difference: $11.42 \pm 36.00 ; 95 \%$ CI: -68.79 to $91.63 ; t_{10}=0.3172, P=0.7576$ ), $n=6$ mice per genotype. c-e, Representative images of Fos induction (cyan) in the mPFC (c), dHPC (d) and BLA (e, outlined in dashed lines) of Cre-negative and Myrf icKO animals following 30-day retrieval. f, Quantification of $\mathrm{Fos}^{+}$density across brain regions following 24-h retrieval sessions. Unpaired two-tailed $t$-tests, mPFC (difference: $-18.95 \pm 25.61$; $95 \%$ CI: -76.90 to $38.99 ; t_{9}=0.74, P=0.4781$ ), BLA (difference: $41.33 \pm 39.72 ; 95 \%$ CI: -48.53 to $131.2 ; t_{9}=1.041, P=0.3252$ ), DG (difference: $64.80 \pm 111.0 ; 95 \% \mathrm{CI}:-186.2$ to $315.8 ; t_{9}=0.5839, P=0.5736$ ), CA3 (difference: $-45.58 \pm 123.5 ; 95 \%$ CI: -324.9 to 233.7 ; $\left.t_{9}=0.3692, P=0.7205\right), n=5$ Cre-negative and 6 Myrf icKO mice. $\mathbf{g}-\mathbf{j}$, Individual 30-day freezing responses plotted against Fos ${ }^{+}$cell density in the $\operatorname{mPFC}(\mathbf{g})$, the BLA $(\mathbf{h})$, the DG (i) and the CA3 (j). Two-tailed Spearman's correlation test, $\mathrm{mPFC}\left(R_{\mathrm{S}}=0.589 ; 95 \%\right.$ CI: 0.1853 to $0.8231 ; P=0.0062)$, BLA $\left(R_{\mathrm{S}}=0.467 ; 95 \%\right.$ CI: 0.04322 to $0.7483 ; P=0.0284$ ), DG $\left(R_{\mathrm{S}}=0.451 ; 95 \%\right.$ CI: 0.03545 to $\left.0.7343 ; P=0.305\right)$, CA3 $\left(R_{\mathrm{S}}=0.535 ; 95 \%\right.$ CI: 0.1449 to $0.7812 ; P=0.0085), n=23$ mice. Scale bars, $200 \mu \mathrm{m}(\mathbf{c}-\mathbf{e})$. Data are presented as the mean \pm s.e.m. $* P \leq 0.05, * * P \leq 0.01$. 

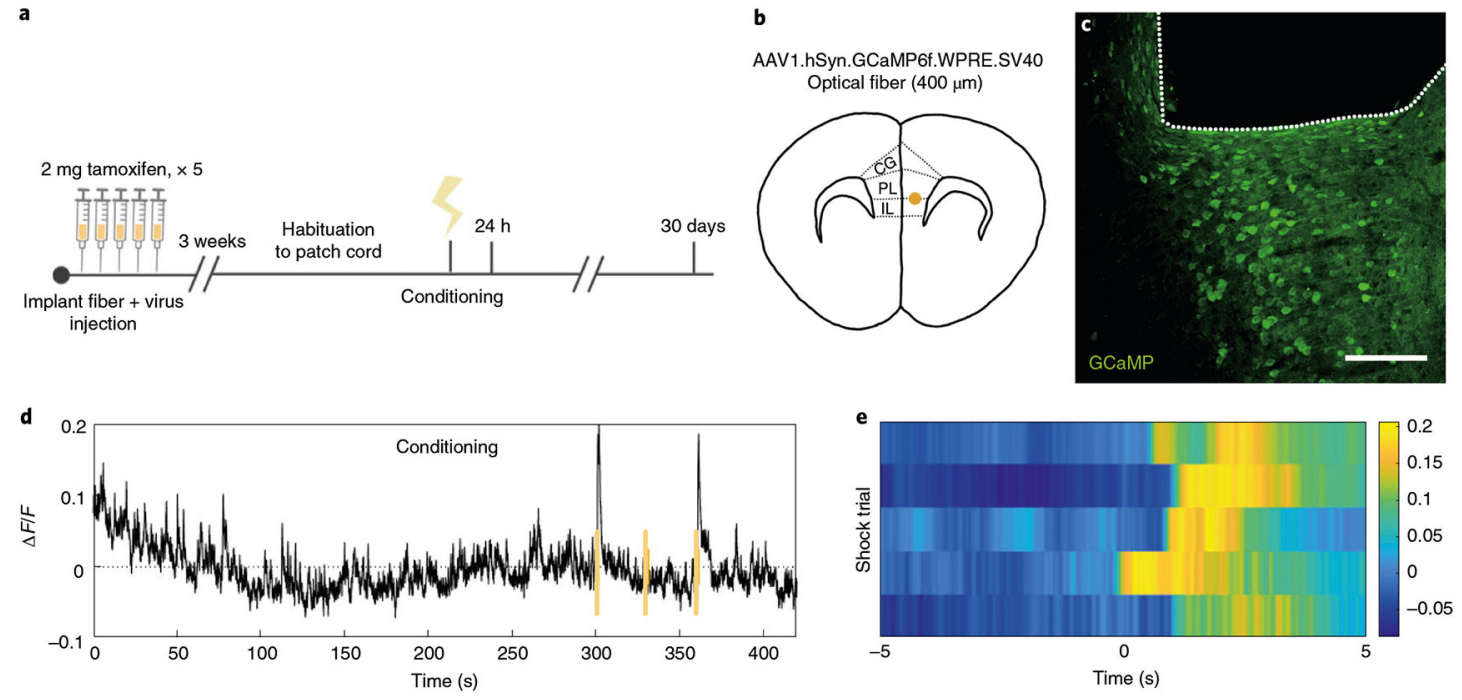

f
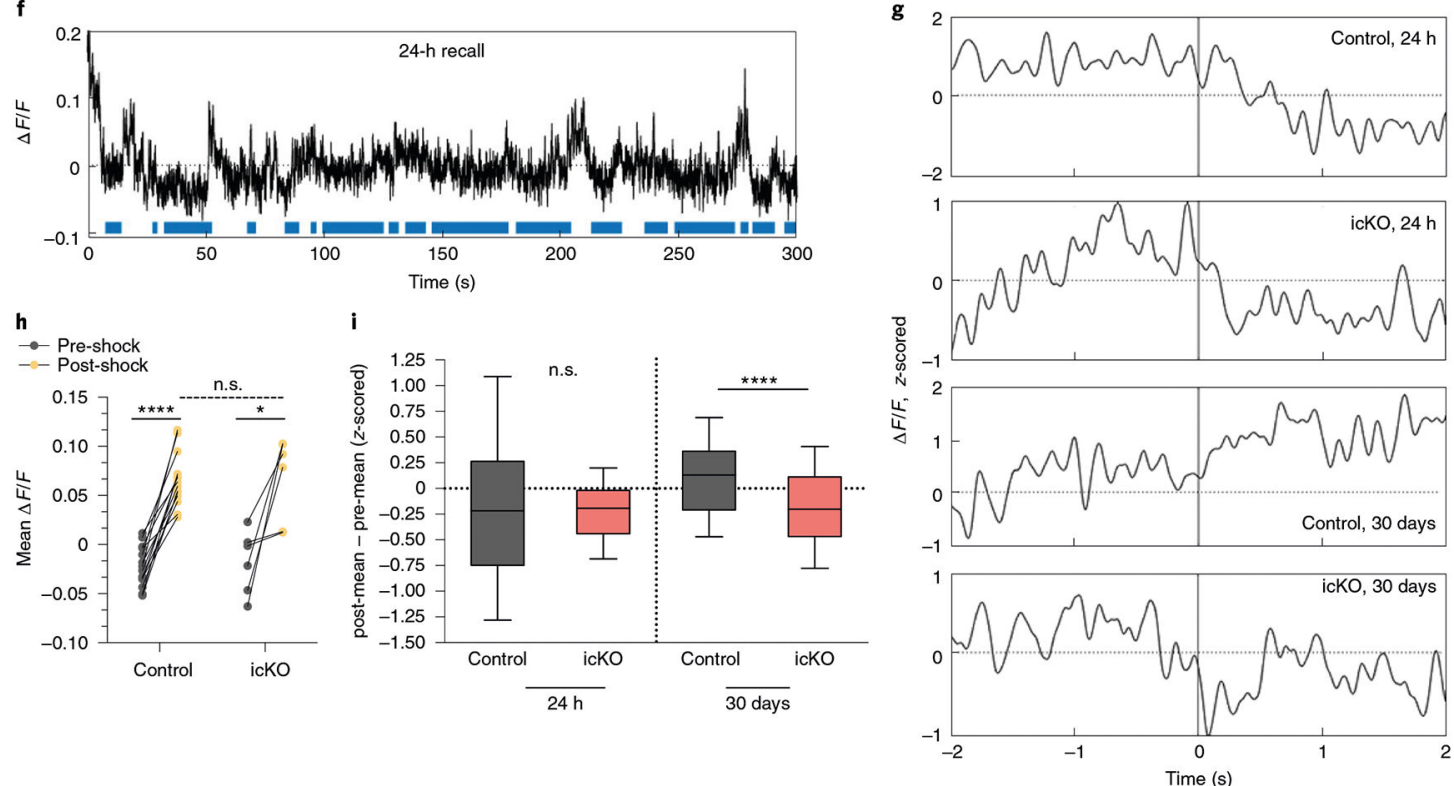

Fig. 6 |. Prefrontal population calcium dynamics in the $\mathrm{mPFC}$ are altered in the absence of new myelin formation.

a, Experimental paradigm for surgeries, tamoxifen administration and fiber photometry recordings during contextual fear conditioning and retrieval. b,c, Schematic and anatomical location (CG, cingulate gyrus) (b) for delivery of calcium indicator and optical fiber and representative image (c) of GCaMP expression (green) and fiber placement (outlined by dashed line) in the mPFC. $\mathbf{d}$, Representative $\Delta F F$ trace across a conditioning session, with foot shocks overlaid in yellow. e, Heatmap of $\Delta F / F$ values $5 \mathrm{~s}$ pre- and post-shock for the first shock delivered in five representative animals; color map represents the $\triangle F / F$. $\mathbf{f}$, Representative $\Delta F / F$ trace across a 24 -h retrieval session with freezing overlaid in blue. $\mathbf{g}$, Representative $Z$-scored $\Delta F / F$ traces across a time window spanning $2 \mathrm{~s}$ pre- and post-bout transition. h, Quantification of the mean $\Delta F / F$ during the $5 \mathrm{~s}$ pre- and post-shock, stratified by genotype; $n=14$ bouts per 7 mice (control) and 6 bouts per 3 mice (Myrf icKO). Paired two-tailed $t$-tests comparing pre- and post-shock for control (difference: $0.08767 \pm$ 
0.007947 ; $95 \%$ CI: 0.07051 to $0.1048 ; t_{13}=11.03, P<0.0001$ ) and $M y r f$ icKO (difference: $0.08468 \pm 0.02609 ; 95 \%$ CI: 0.01760 to $\left.0.1518 ; t_{5}=3.245, P=0.0031\right)$ animals. Unpaired two-tailed $t$-test comparing post-shock across genotypes (difference: $0.001432 \pm 0.01574$; 95\% CI: -0.03163 to $\left.0.03449 ; t_{18}=0.09098, P=0.9285\right)$. i, Quantification of the difference between the $z$-scored mean $\Delta F / F$ of the $2 \mathrm{~s}$ pre- and post-bout transition for control and $M y r f$ icKO animals, plotted during 24-h (left bars) and 30-day (right bars) retrieval; $n=10$ bouts for 7 mice (control) and 7 mice ( $M y r f$ icKO). Unpaired two-tailed $t$-tests comparing across genotypes at $24 \mathrm{~h}$ (difference $-0.02001 \pm 0.1516 ; 95 \% \mathrm{CI}:-0.3192$ to $0.2792 ; t_{138}=0.132$, $P=0.8951$ ) and 30 days (difference: $-0.2963 \pm 0.07301 ; 95 \%$ CI: -0.4403 to $-0.1524 ; t_{138}$ $=4.5059, P<0.0001)$. Scale bar, $100 \mu \mathrm{m}$. For box-and-whisker plots, the center, boxes and whiskers represent the median, interquartile range, and the 10th and 90 th percentiles. n.s., not significant, $P>0.05, * * P \leq 0.01, * * * * P \leq 0.0001$. 

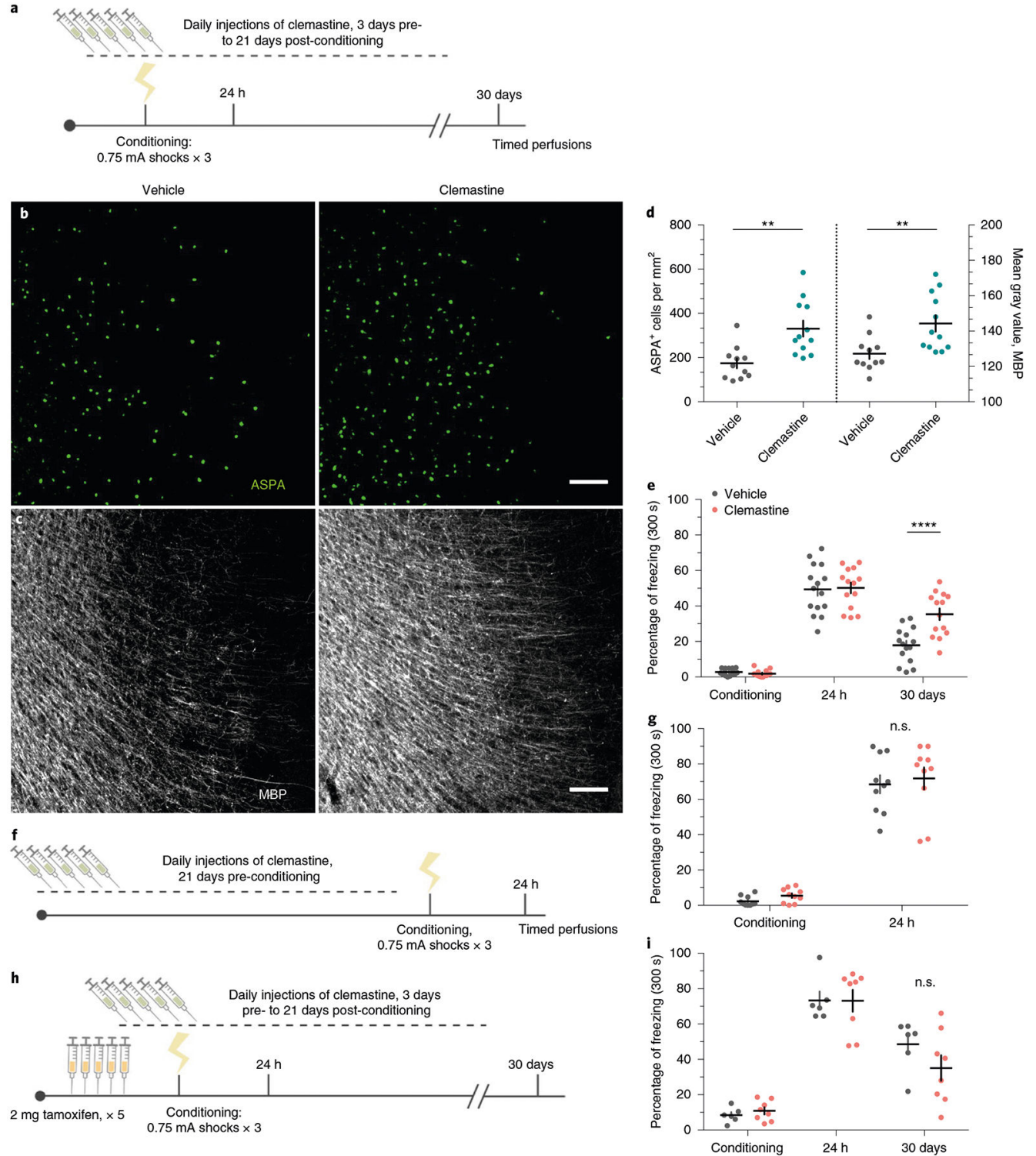

Fig. 7 |. induction of new myelin formation preserves remote fear memory recall.

a, Experimental paradigm for clemastine injections and contextual fear conditioning. b,c, Representative images of ASPA (green) and MBP (gray) signal in the MPFC of vehicletreated mice (b) and clemastine-treated mice (c) 30 days post-conditioning. d, Quantification of $\mathrm{ASPA}^{+}$cell density (left bars) and MBP mean fluorescence (right bars) in the mPFC of vehicle-and clemastine-treated animals, Unpaired two-tailed $t$-tests comparing vehicle versus clemastine for $\mathrm{ASPA}^{+}$density (difference: $156.9 \pm 43.21$; 95\% CI: 67.00 to $246.7 ; t_{21}=$ 3.63, $P=0.0016$ ) and MBP fluorescence (difference: $17.10 \pm 5.640 ; 95 \%$ CI: 5.370 to $\left.28.83 ; t_{21}=3.032, P=0.0063\right), n=11$ vehicle-treated and 12 clemastine-treated mice. $\mathbf{e}$, Individual fear expression over time for vehicle-treated mice and clemastine-treated mice. Two-way ANOVA $(F 4,108=5.884, P=0.0003)$ with Sidak's post hoc tests comparing across treatment groups for conditioning (difference: $0.8941 \pm 4.116$; $95 \%$ CI: -9.860 to $11.65 ; P=0.9928), 24$ h (difference: $-0.8004 \pm 4.116$; $95 \%$ CI: -11.55 to $9.954 ; 0.9948$ ) 
and 30 days (difference: $-17.49 \pm 4.116 ; 95 \%$ CI: -16.00 to $-0.3832 ; P=0.0002$ ), $n=15$ vehicle-treated and 14 clemastine-treated mice. f, Experimental timeline controlling for the duration of clemastine injections, in which the entire injection protocol is completed before initiation of fear conditioning and recent memory recall; $n=10$ mice per treatment. $\mathbf{g}$, Individual freezing responses during recent memory recall testing for animals pretreated with clemastine in the experimental timeline shown in $\mathbf{f}$. Two-way ANOVA $\left(F_{1,18}=\right.$ 0.0003928, $P=0.9844)$. h, Experimental paradigm for clemastine injections in Myrf icKO animals. $\mathbf{i}$, Individual fear expression over time for vehicle-treated mice and clemastinetreated Myrf icKO mice. Two-way ANOVA $\left(F_{4,48}=1.357, P=0.2629\right), n=6$ vehicletreated and 8 clemastine-treated mice. Scale bars, $100 \mu \mathrm{m}(\mathbf{b}, \mathbf{c})$. Data are presented as the mean \pm s.e.m. $* * P \leq 0.01, * * * * P \leq 0.0001$. 


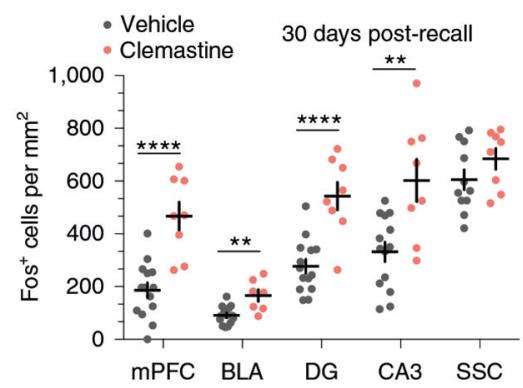

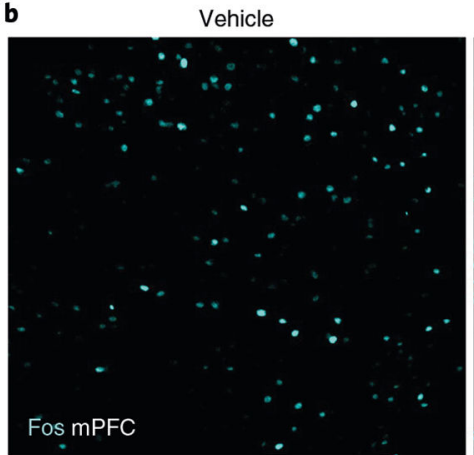

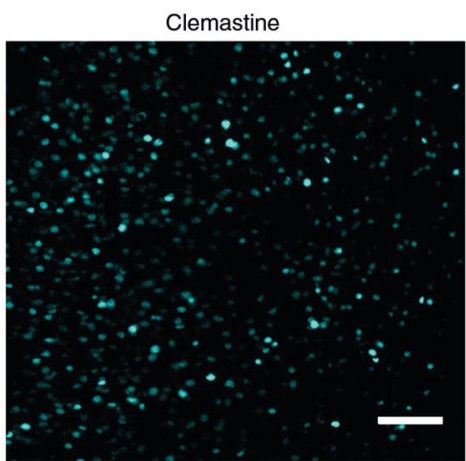

Clemastine

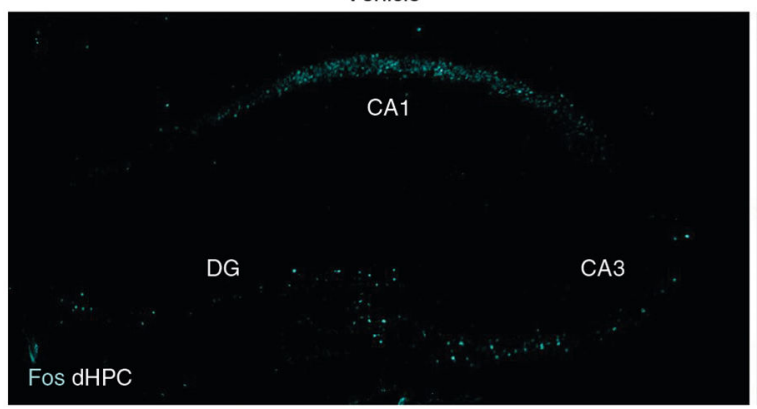

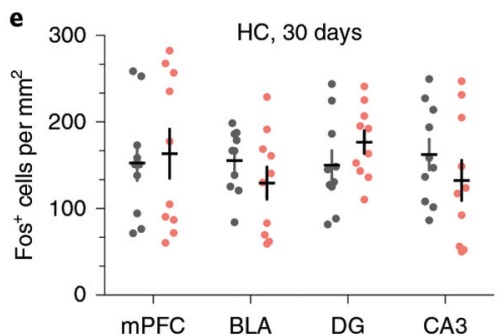

Fig. 8 | induction of new myelin formation increases immediate early gene expression following remote fear memory recall.

a, Quantification of $\mathrm{Fos}^{+}$cell density across brain regions in vehicle-treated mice and clemastine-treated mice following 30-day retrieval. Unpaired two-tailed $t$-tests, mPFC (difference: $280.7 \pm 54.50 ; 95 \%$ CI: 167.0 to $394.4 ; t_{19}=5.15, P<0.0001$ ), BLA (difference: $75.06 \pm 20.12 ; 95 \% \mathrm{CI}: 32.95$ to $117.2 ; t_{19}=3.73, P=0.0014$ ), DG (difference: $268.7 \pm 56.69 ; 95 \%$ CI: 150.0 to $387.3 ; t_{19}=4.739, P<0.0001$ ), CA3 (difference: $270.5 \pm$ 77.34; $95 \%$ CI: 109.2 to $431.8, t_{19}=3.497 ; P=0.0023$ ), SSC (difference: $79.25 \pm 55.73$; 95\% CI: -38.33 to $\left.196.8 ; t_{19}=1.422, P=0.1731\right), n=13$ vehicle-treated and 8 clemastinetreated mice. b,c, Representative images of Fos induction (cyan) in the mPFC (b) and dHPC (c) of vehicle- and clemastine-treated animals following 30-day retrieval. d, Quantification of $\mathrm{Fos}^{+}$cell density across brain regions in vehicle-pretreated mice and clemastinepretreated mice, represented in Fig. 7f,g, following 24-h retrieval. Unpaired two-tailed $t$ tests, mPFC (difference: $6.885 \pm 35.98 ; 95 \% \mathrm{CI}:-68.71$ to $82.48 ; t_{18}=0.1913, P=0.8504$ ), BLA (difference: $-22.53 \pm 17.11 ; 95 \% \mathrm{CI}:-58.48$ to $13.42 ; t_{18}=1.317, P=0.2045$ ), DG (difference: $3.547 \pm 41.64 ; 95 \% \mathrm{CI}:-83.94$ to $91.04 ; t_{18}=0.08518, P=0.9331$ ), CA3 (difference: $5.178 \pm 33.28 ; 95 \% \mathrm{CI}$ : -64.74 to $75.10 ; t_{18}=0.1556, P=0.8781$ ), $n=10$ mice per treatment group. e, Quantification of $\mathrm{Fos}^{+}$cell density across brain regions in $\mathrm{HC}$ 
vehicle-treated animals and clemastine-treated animals, represented in Extended Data Fig. $6 \mathrm{a}, \mathrm{g}$, in the absence of fear learning. Unpaired two-tailed $t$-tests, mPFC (difference: $10.88 \pm$ 35.04; 95\% CI: -62.73 to $84.50 ; t_{18}=0.3106, P=0.7597$ ), BLA (difference: $-25.98 \pm$ 22.03; 95\% CI: -72.27 to $20.31 ; t_{18}=1.179, P=0.2537$ ), DG (difference: $26.58 \pm 21.51$; 95\% CI: -18.61 to $71.77 ; t_{18}=1.236, P=0.2324$ ), CA3 (difference: $-29.91 \pm 29.50$; $95 \%$ CI: -91.88 to $\left.32.06 ; t_{18}=1.014, P=0.3239\right), n=10$ mice per treatment group. Scale bars, $100 \mu \mathrm{m}(\mathbf{b})$ or $200 \mu \mathrm{m}(\mathbf{c})$. Data are presented as the mean \pm s.e.m. $* * P \leq 0.01$, $* * * * P \leq$ 0.0001 . 